\title{
MYC paralog-dependent apoptotic priming orchestrates a spectrum of vulnerabilities in small cell lung cancer
}

Marcel A. Dammert1,2,3,14, Johannes Brägelmann1,2,3,4,14, Rachelle R. Olsen ${ }^{5,14}$, Stefanie Böhm²,3,14, Niloufar Monhasery 1,2,3, Christopher P. Whitney ${ }^{5}$, Milind D. Chalishazar ${ }^{5}$, Hannah L. Tumbrink ${ }^{1,2,3}$, Matthew R. Guthrie ${ }^{5}$, Sebastian Klein 2,3,4,6, Abbie S. Ireland ${ }^{5}$, Jeremy Ryan (1) 7, Anna Schmitt8,9, Annika Marx ${ }^{1,2,3}$, Luka Ozretić ${ }^{10}$, Roberta Castiglione ${ }^{4,6}$, Carina Lorenz ${ }^{1,2,3}$, Ron D. Jachimowicz ${ }^{8,9}$, Elmar Wolf (1) ${ }^{11}$, Roman K. Thomas ${ }^{2}$, John T. Poirier (10 ${ }^{12}$, Reinhard Büttner ${ }^{6}$, Triparna Sen ${ }^{13}$, Lauren A. Byers ${ }^{13}$, H. Christian Reinhardt ${ }^{4,8,9}$, Anthony Letai (i) ${ }^{7}$, Trudy G. Oliver (iD ${ }^{5} \&$ Martin L. Sos ${ }^{1,2,3}$

MYC paralogs are frequently activated in small cell lung cancer (SCLC) but represent poor drug targets. Thus, a detailed mapping of MYC-paralog-specific vulnerabilities may help to develop effective therapies for SCLC patients. Using a unique cellular CRISPR activation model, we uncover that, in contrast to $M Y C N$ and $M Y C L, M Y C$ represses $B C L 2$ transcription via interaction with MIZ1 and DNMT3a. The resulting lack of BCL2 expression promotes sensitivity to cell cycle control inhibition and dependency on MCL1. Furthermore, MYC activation leads to heightened apoptotic priming, intrinsic genotoxic stress and susceptibility to DNA damage checkpoint inhibitors. Finally, combined AURK and CHK1 inhibition substantially prolongs the survival of mice bearing MYC-driven SCLC beyond that of combination chemotherapy. These analyses uncover MYC-paralog-specific regulation of the apoptotic machinery with implications for genotype-based selection of targeted therapeutics in SCLC patients.

\footnotetext{
${ }^{1}$ Molecular Pathology, Institute of Pathology, University Hospital of Cologne, 50937 Cologne, Germany. ${ }^{2}$ Department of Translational Genomics, Center of Integrated Oncology Cologne-Bonn, Medical Faculty, University of Cologne, 50931 Cologne, Germany. ${ }^{3}$ Center for Molecular Medicine Cologne, University of Cologne, 50931 Cologne, Germany. ${ }^{4}$ Else Kröner Forschungskolleg Clonal Evolution in Cancer, University Hospital Cologne, 50931 Cologne, Germany. ${ }^{5}$ Department of Oncological Sciences, Huntsman Cancer Institute, University of Utah, Salt Lake City, UT 84112, USA. ${ }^{6}$ Institute of Pathology, University Hospital of Cologne, 50937 Cologne, Germany. ${ }^{7}$ Dana-Farber Cancer Institute, Harvard Medical School, Boston, MA 02215, USA. ${ }^{8}$ Department I of Internal Medicine, University Hospital of Cologne, 50931 Cologne, Germany. ${ }^{9}$ Cologne Excellence Cluster on Cellular Stress Response in Aging-Associated Diseases, University of Cologne, 50931 Cologne, Germany. ${ }^{10}$ Department of Cellular Pathology, Royal Free Hospital, London NW3 2QG, UK. ${ }^{11}$ Theodor Boveri Institute, Biocenter, University of Würzburg, 97074 Würzburg, Germany. ${ }^{12}$ Memorial Sloan Kettering Cancer Center, New York, NY 10065, USA. ${ }^{13}$ Department of Thoracic and Head \& Neck Medical Oncology, University of Texas, MD Anderson Cancer Center, Houston, TX 77030, USA. ${ }^{14}$ These authors contributed equally: Marcel A. Dammert, Johannes Brägelmann, Rachelle R. Olsen, Stefanie Böhm. Correspondence and requests for materials should be addressed to T.G.O. (email: trudy.oliver@hci.utah.edu) or to M.L.S. (email: martin.sos@uni-koeln.de)
} 
S mall cell lung cancer (SCLC) is an aggressive neuroendocrine subtype of lung cancer with a 5-year survival rate of only $6 \%$ that lacks effective targeted therapies or predictive markers for patient stratification. Genomic amplification of one of the transcription factor paralogs $M Y C, M Y C N$, or $M Y C L$ occurs in approximately $20 \%$ of SCLC patients ${ }^{1,2}$. MYC paralog activation is important for tumorigenesis and tumor maintenance, which would make MYC an ideal target for therapeutic intervention $^{3-5}$. While direct inhibition of MYC has not yet been achieved, MYC paralog activation in SCLC induces distinct sensitivity profiles to targeted agents such as Aurora Kinase (AURK) or DNA damage checkpoint inhibitors that are preferentially effective in $M Y C$-activated cells ${ }^{6-9}$. At the same time, $\mathrm{BH} 3$ mimetics, including drugs directed against the anti-apoptotic factors BCL2 and MCL1, represent an attractive class of inhibitors in SCLC but it remains unclear which molecular factors prime susceptibility to these targets ${ }^{10}$. How overexpression of the individual MYC paralogs shapes the spectrum of vulnerabilities in SCLC remains elusive.

We hypothesize that a mechanistic understanding of the phenotypic differences associated with activation of individual MYC paralogs may allow the discovery of molecularly defined drug targets in SCLC patients. Using CRISPR/dCas9-mediated MYC paralog activation, we uncover a link between MYC signaling and the regulation of the apoptotic machinery with direct implications for the selection of targeted drugs for SCLC patients.

\section{Results}

MYC activation is associated with low BCL2 expression. We analyzed transcriptomes of 42 patient-derived SCLC cell lines and 81 SCLC patient samples ${ }^{1,6,11}$ and found that overexpression of individual MYC paralogs is largely mutually exclusive in both datasets (Fig. 1a, b). At the same time, the impact of individual MYC paralogs on overall survival remains unclear due to the limited amount of available expression data in SCLC patient cohorts (Supplementary Fig. 1a) ${ }^{12}$. These observations prompted us to dissect the specific role of each MYC paralog in SCLC, with the CRISPR/dCas9 Synergistic Activation Mediator (SAM) CRISPR activation (CRISPRa) system ${ }^{13}$ that allows efficient induction of endogenous gene expression. After single guide RNA (sgRNA) selection and validation in NIH3T3 and GEMM-derived (Trp53/Rb1-deficient mice, RP) mouse embryonic fibroblasts (MEFs), we activated $M y c, M y c n$, or $M y c l$ in genomically profiled (whole-exome sequencing (WES)) cells derived from early stage SCLC (RP) tumors ${ }^{14}$ (Supplementary Fig. 1b-d). We observed increased transcription of the individual $M y c$ paralogs and elevated MYC and MYCN protein expression (Fig. 1c, d). Although the magnitude of upregulation differed among $M y c$ paralogs (Fig. 1c and Supplementary Fig. 1b, c), canonical MYC target genes $^{6}$ were similarly upregulated and proliferation rates were similar between individual cells (Fig. 1c and Supplementary Fig. 1e). However, $M y c$ - but not $M y c n$ - or $M y c l$-activation induced sensitivity to the AURK inhibitor, alisertib (Fig. 1e), and other cell cycle checkpoint inhibitors (volasertib, $p=0.006$ mock vs. $M y c$; adavosertib, $p=0.05$ mock vs. $M y c$, two-tailed unpaired $t$ test) similar to patient-derived SCLC cells ${ }^{6,7}$ (Supplementary Fig. 1f).

We next determined differentially expressed genes in MYChigh $(n=22)$ vs. MYC-low $(n=20)$ human SCLC cell lines (Fig. 1a) $)^{6,7}$ to investigate these $M Y C$-specific vulnerabilities. Consistent with the $M Y C$-associated variant SCLC phenotype, high MYC expression correlated with elevated NEUROD1 (Fig. 1f) ${ }^{6}$. Intriguingly, anti-apoptotic factor BCL2 was significantly downregulated in $M Y C$-overexpressing cells while other BCL2 family members were not differentially expressed (Fig. If and Supplementary Fig. 1g, h). In addition, we observed a modest trend toward a negative correlation of $M Y C$ and BCL2 in an independent cohort of SCLC patients ${ }^{15}$ (Supplementary Fig. 1i) and significantly decreased $B c l 2$ expression in $M y c$-high tumors of $M y c$-driven SCLC mice (RPM) compared to Trp53/Rb1-deficient SCLC mouse tumors with low $M y c$ expression (Supplementary Fig. $1 \mathrm{j})^{6}$. Furthermore, BCL2 and ASCL1 proteins were only expressed in $M Y C N$ - and MYCL-amplified cells (Fig. $1 \mathrm{~g}$ and Supplementary Fig. 1k). We observed a similar anti-correlation of MYC and BCL2 protein levels in immunohistochemical (IHC) stainings of human SCLC tumor specimens $(n=48)$ (Fig. $1 \mathrm{~h}$ and Supplementary Table 1). Myc activation also suppressed $B c l 2$ expression in CRISPRa cells $(p=0.004$ mock vs. $M y c$, two-tailed unpaired $t$ test) (Fig. 1i). This anti-correlation between MYC and BCL2 appears to be an exception rather than the rule since we primarily found a positive correlation between MYC and BCL2 expression in the pan-cancer CCLE cohort ${ }^{16,17}$ (Supplementary Fig. 11). Reintroduction of BCL2 strongly reduced sensitivity toward alisertib in both $M Y C$-amplified patient-derived cell lines (Fig. 1j, k) and $M y c$-activated CRISPRa cells (Fig. 1l, m). Conversely, co-treatment of BCL2-overexpressing $M y c$-activated CRISPRa cells with BCL2-specific inhibitor venetoclax restored the activity of alisertib (Fig. $1 \mathrm{~m}$ ). Of note, exogenous BCL2 overexpression did not alter cell cycle progression or proliferation rates (Supplementary Fig. 1m, n). Thus MYC paralog expression is tightly linked with BCL2 expression, which determines susceptibility to cell cycle checkpoint inhibitors.

MYC represses $B C L 2$ expression. As reported previously ${ }^{10}$, $B C L 2$ expression only partially translated into BCL2 inhibitor activity (Fig. 2a, b and Supplementary Fig. 2a-d). Patient-derived $(n=4)$ and murine CRISPRa cell lines with MYCN/Mycn overexpression were sensitive to BCL2 inhibitors navitoclax and ABT737, whereas $M Y C / M y c$-overexpressing cells were more resistant to BCL2 inhibition (Fig. 2a, b and Supplementary Fig. 2a-d). Since the CRISPRa cells showed an adherent growth phenotype that is associated with basal activation of $M y c$ in these cells ${ }^{6,18}$, we performed short hairpin RNA (shRNA)-mediated knockdown of the endogenous $M y c$ in $M y c n$-activated CRISPRa cells (Fig. 2c and Supplementary Fig. 2e). Myc knockdown induced Bcl2 expression (Fig. 2c) and increased sensitivity to BCL2 inhibitors (Fig. 2d, e and Supplementary Fig. 2f, g). Since repression of BCL2 correlates with high DNA methylation at the BCL2 promoter $^{19}$, we assayed DNA methylation levels of the CpG island within the BCL2 promoter in human SCLC cell lines. MYC-amplified cell lines $(n=3)$ displayed high DNA methylation levels at the BCL2 promoter (Fig. 2f), whereas $M Y C N$ - or $M Y C L$-amplified cells $(n=3)$ exhibited significantly less DNA methylation in this region indicating active transcription $(M Y C$-amplified vs. non$M Y C$-amplified $p=0.0001$, two-tailed unpaired $t$ test; Fig. 2g). Similarly, high MYC expression correlated with high levels of BCL2 promoter methylation in published methylation data of SCLC cell lines $(n=65)$ (Supplementary Fig. $2 \mathrm{~h})^{20}$ and patientderived xenograft SCLC models (Supplementary Fig. $2 \mathrm{i}, \mathrm{j})^{21}$. These observations implicate a functional link between high $M Y C$ expression, increased BCL2 promoter methylation, and low BCL2 expression.

MYC was shown to facilitate the establishment of DNA methylation at gene promoters by cooperating with MIZ1 and DNA methyltransferase 3a (DNMT3a) ${ }^{22}$. Using chromatin immunoprecipitation (ChIP) assays, we observed co-occupancy of MYC, MIZ1, and DNMT3a at the BCL2 promoter (Fig. 2h) with MYC binding at the transcriptionally inactive BCL2 promoter being as pronounced as at the active $A C T B$ promoter. MIZ1 and DNMT3a were enriched only at the BCL2 promoter in MYC-high cells (Fig. 2h). This suggests that MYC/MIZ1/ 
a

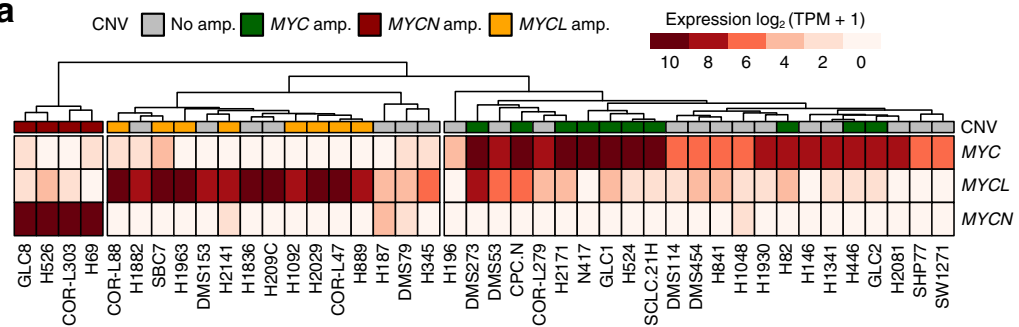

C

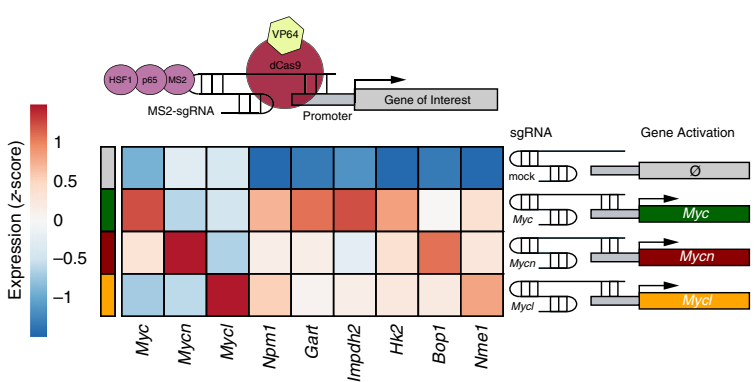

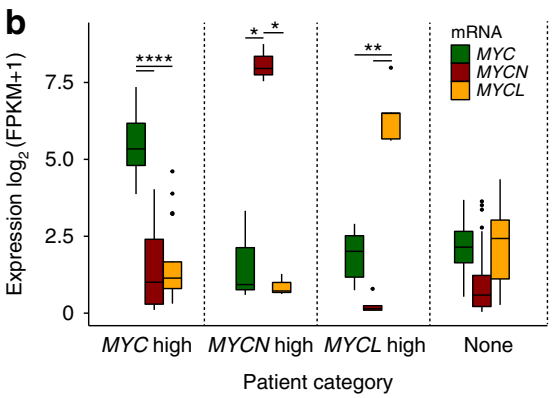

d
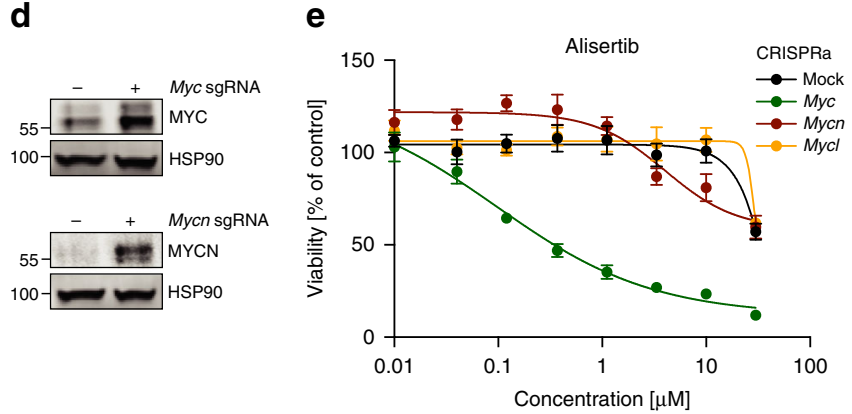

f

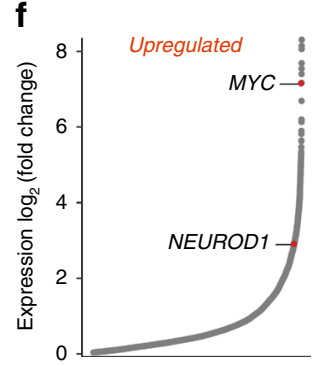

i

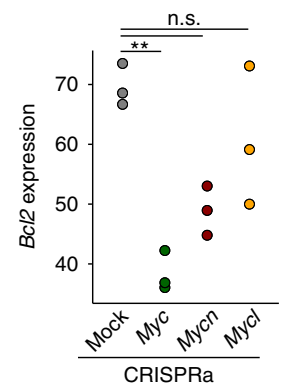

g
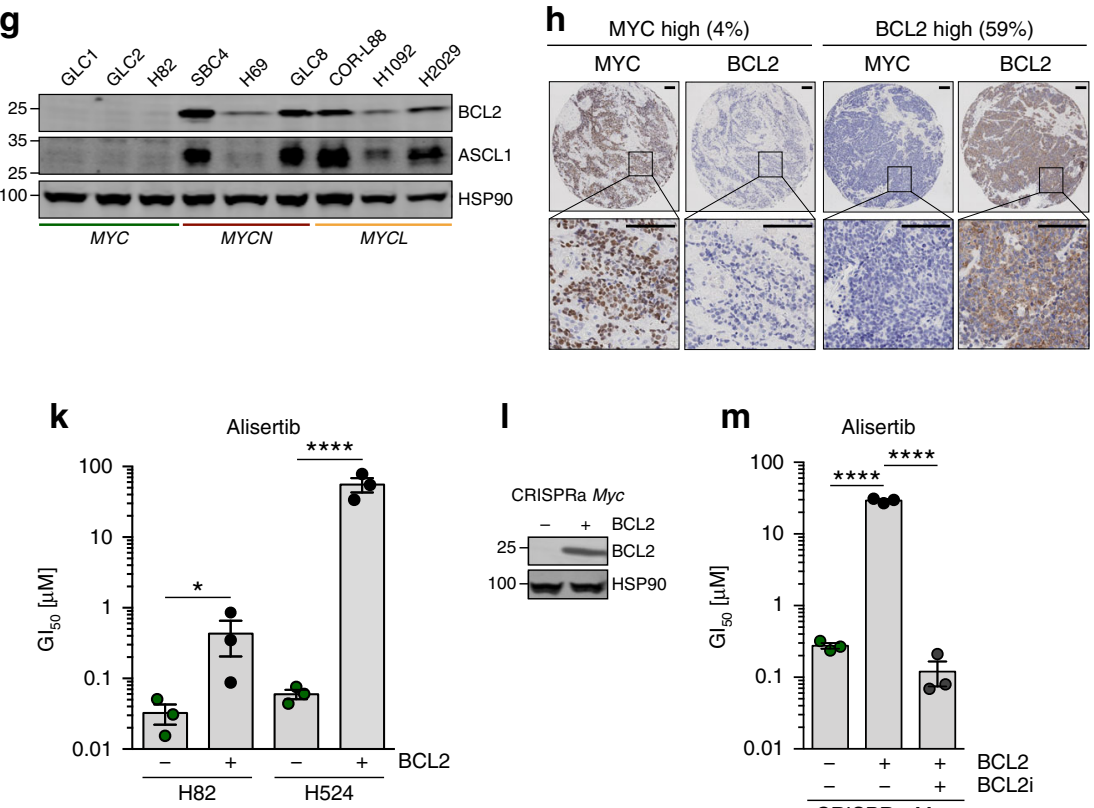

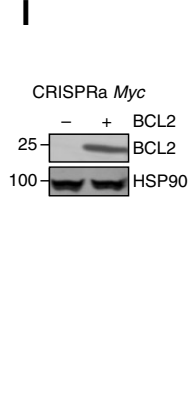

Fig. 1 MYC activation is associated with low BCL2 expression. a MYC paralog expression (TPM) and copy number variation (CNV) in human small cell lung cancer (SCLC) cell lines $(n=42)$. b MYC paralog expression in SCLC patients. Center line (median), lower/upper box hinges (25th/75th percentile), whiskers extend to the most extreme value within $1.5 \times$ interquartile range (IQR) of the hinges. c CRISPRa system for transcriptional upregulation of Myc paralogs (top). Expression (z-scores) of Myc paralogs and Myc target genes in CRISPRa cells (bottom). $\mathbf{d}$ Western blot showing MYC and MYCN in Mycand Mycn-activated CRISPRa cells compared to mock control. HSP9O was used as a loading control. e Viability screening of Myc-activated CRISPRa cells treated with alisertib for $96 \mathrm{~h}(n=3)$. $\mathbf{f}$ Differentially upregulated and downregulated genes (sorted by $\log _{2}$ fold-change) in human SCLC cell lines ( $n=42$ ) with high MYC ( $n=22)$ vs. low MYC $(n=20)$ expression. $\mathbf{g}$ Western blot of ASCL1 and BCL2 in MYC paralog-amplified human SCLC cell lines ( $n=9)$. HSP90 was used as a loading control. h Immunohistochemical staining of MYC and BCL2 in tumors of SCLC patients with high MYC (left) or high BCL2 (right) expression (percentage of patients in the cohort $(n=49)$ with high BCL2 or MYC levels is indicated. Scale bar $=100 \mu \mathrm{m}$ ). i $B c / 2$ expression (counts normalized to library size) in Myc paralog-activated CRISPRa cells. Benjamini-Hochberg-adjusted $p$ values for Myc paralogs were obtained as contrasts of a global differential expression test. j Western blot showing BCL2 levels in MYC-amplified H82 and H524 cells \pm BCL2 overexpression. HSP90 was used as a loading control. $\mathbf{k ~ G l} 50$ values of $M Y C$-amplified $\mathrm{H} 82$ and $\mathrm{H} 524$ cells $\pm B C L 2$ overexpression treated with alisertib for $72 \mathrm{~h}(n=3)$. I Western blot of BCL2 in Myc-activated CRISPRa cells $\pm B C L 2$ overexpression. HSP90 was used as a loading control. $\mathbf{m}$ GI 50 values of Myc-activated CRISPRa cells $\pm B C L 2$ overexpression treated with alisertib alone or in combination with $500 \mathrm{nM}$ venetoclax $(B C L 2 i ; n=3)$. Error bars indicate mean \pm SEM. Two-tailed unpaired $t$ tests, ${ }^{\star \star \star *} p<0.0001,{ }^{\star \star} p<0.01,{ }^{\star} p<0.05$. Source data are provided as a Source Data file 

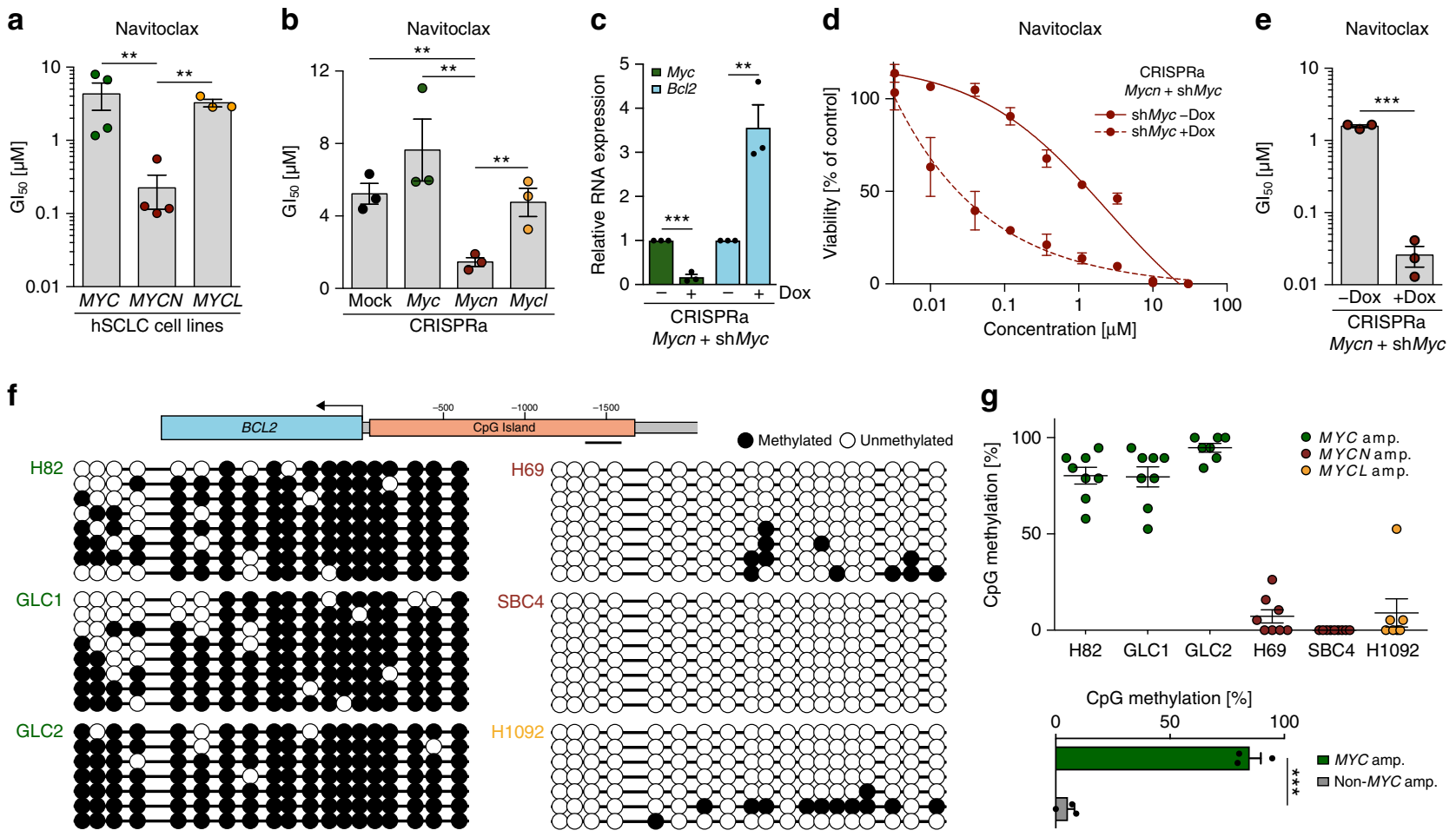

h

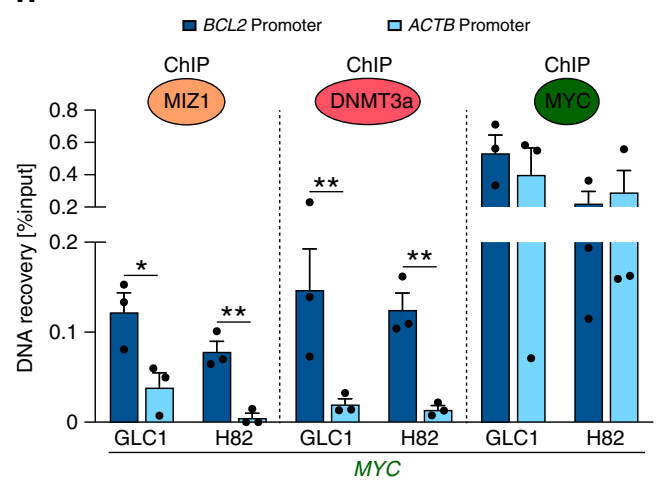

i

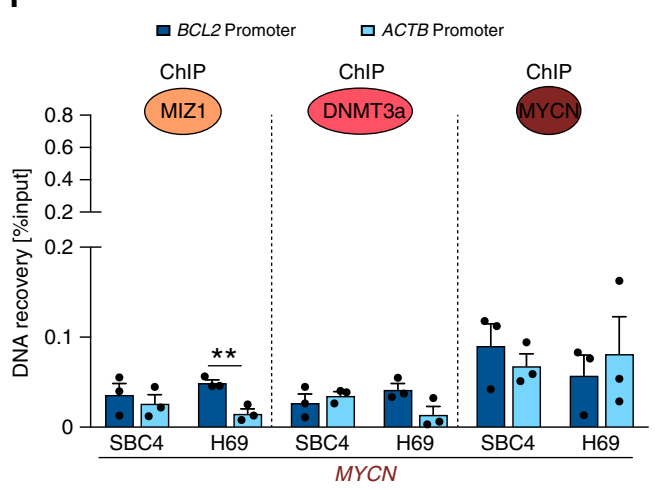

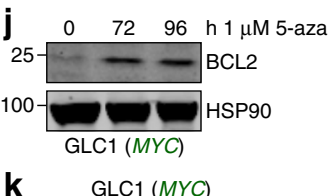

k

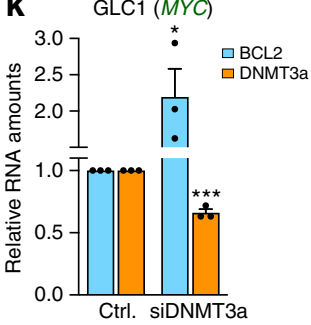

Fig. 2 MYC represses BCL2 expression. a GI 50 values of $M Y C(n=4 ; \mathrm{GLC1}, \mathrm{H} 82, \mathrm{H} 524, \mathrm{GLC} 2), \mathrm{MYCN}(n=4$; GLC8, H69, SBC4, COR-L303), and MYCL ( $n=3$; H1092, H2029, SBC7) human small cell lung cancer (SCLC) cell lines treated with navitoclax for $72 \mathrm{~h}(n=3)$. b Gl 50 values of Myc paralogactivated CRISPRa cells treated with navitoclax for $96 \mathrm{~h}(n=3)$. c Relative mRNA levels (quantitative reverse transcription polymerase chain reaction ( $\mathrm{qRT}$ P(R)) of Myc and Bc/2 in Mycn-activated CRISPRa cells with Tet-inducible Myc knockdown by shRNA at $72 \mathrm{~h}$ after doxycycline treatment $(n=3)$. Data were normalized to $18 \mathrm{~S}$ rRNA. d Viability screening of Mycn-activated CRISPRa cells after Myc knockdown treated with navitoclax for $96 \mathrm{~h}(n=3)$. e GI 50 values of viability screening in $\mathbf{d}(n=3)$. $\mathbf{f}$ DNA methylation at the BCL2 promoter (indicated region) measured by bisulfite sequencing. Lollipop diagram representing methylated (black) and unmethylated (white) CpGs in MYC (H82: $n=8, \mathrm{GLC1}: n=8, \mathrm{GLC2}: n=7), M Y C N(H 69: n=8, S B C 4: n=8)$, and MYCL (H1092: $n=7$ ) amplified human SCLC cell lines. $g$ Percentage of methylated CpG residues in MYC (H82, GLC1, GLC2), MYCN (H69, SBC4), and MYCL (H1092) amplified human SCLC cell lines (top). CpG methylation percentage (f) in SCLC cell lines $(n=6)$ grouped by MYC amplification status (MYC-high $n=3$, MYC-low $n=3$; bottom). h, i Occupancy at the BCL2 promoter of MYC, MIZ1, and DNMT3a in MYC-amplified cells (GLC1, H82) (h) and of MYCN, MIZ1, and DNMT3a in MYCN-amplified cells (SBC4, H69) (i) measured by chromatin immunoprecipitation (ChIP) quantitative real-time PCR $(n=3)$. ChIP signal is displayed as percentage of input. IgG (non-specific antibody control) signal was subtracted from ChIP signal of specific antibodies. j Western blot showing BCL2 in MYC-amplified GLC1 cells treated with $1 \mu \mathrm{M} 5$-azacytidine for the indicated times. HSP90 was used as loading control. k Relative mRNA expression ( $q R T-P C R$ ) of BCL2 and DNMT3a in MYC-amplified GLC1 cells treated with control small interfering RNA (siRNA) or DNMT3a siRNA $(n=3)$. Data were normalized to $18 \mathrm{~S}$ rRNA. Error bars indicate mean \pm SEM. Two-tailed unpaired $t$ tests, ${ }^{\star \star *} p<0.001,{ }^{\star \star} p<0.01,{ }^{\star} p<0.05$. Source data are provided as a Source Data file

DNMT3a may cooperatively mediate DNA methylation of the $B C L 2$ promoter (Fig. 2f). In contrast, in $M Y C N$-amplified cells only low levels of MIZ1 and DNMT3a were bound to the BCL2 promoter with no enrichment of DNMT3a compared to the $A C T B$ promoter (Fig. 2i). Consistent with previous studies, only MYC but not MYCN or MYCL substantially interacted with
MIZ1 (Supplementary Fig. 2k), which is consistent with the model of MYC-specific BCL2 repression ${ }^{23,24}$. Finally, both pharmacological inhibition of DNA methylation by 5azacytidine in GLC1 cells as well as small interfering RNA (siRNA)-mediated DNMT3a knockdown in two MYC-amplified SCLC cell lines led to de-repression of BCL2 (Fig. 2j, k 
and Supplementary Fig. 2l). Thus DNMT3-mediated DNA methylation may play an important role in the MYC-induced repression of BCL2.

MYC drives apoptotic priming and MCL1 dependency. To assess the impact of differential $B C L 2$ expression on the apoptotic machinery, we performed $\mathrm{BH} 3$ profiling ${ }^{25}$ and observed that MYC-amplified SCLC cell lines $(n=4)$ were more primed for apoptosis induction (Fig. 3a) especially by MS1 peptide, which acts as an MCL1 antagonist (MYC-amplified vs. non-MYCamplified $p=0.01$, two-tailed unpaired $t$ test) (Fig. 3b). Consistently, $M Y C$-amplified SCLC cell lines $(n=4)$ were more sensitive to MCL1 inhibitor $563845^{26}$ compared to MYCN- $(n=$ $3)$ and MYCL- $(n=4)$ amplified cell lines $(p=0.003$ MYC vs. $M Y C N ; p=0.001 M Y C$ vs. MYCL, two-tailed unpaired $t$ test) (Fig. 3c, d and Supplementary Fig. 3a). Despite a lower activity against murine MCL $1^{27}$, we observed an increased susceptibility to S63845 only in Myc but not in Mycn- or Mycl-activated CRISPRa cells (Fig. 3e). We also observed this MYC-induced sensitivity against MCL1 inhibition in clonogenic assays (Supplementary Fig. 3b). In line with previous reports, we observed a reduction of MCL1 protein stability upon AURK inhibition (Supplementary Fig. $3 c)^{28}$ potentially contributing to the high alisertib sensitivity of MYC-overexpressing cells.
BCL2 overexpression mitigated the effects of MCL1 inhibition (Fig. 3f, g), indicating the importance of $M Y C$-induced $B C L 2$ repression in defining MCL1 dependency and MYC-specific vulnerabilities in SCLC. Consistently, siRNA-mediated MCL1 knockdown reduced viability only in $M Y C$-overexpressing cells (Fig. 3h, i) underlining the MYC-induced dependency on MCL1. Interestingly, $M Y C$-amplified cells exhibited increased levels of the DNA-damage response (DDR) marker $\gamma \mathrm{H} 2 \mathrm{AX}$ upon MCL1 knockdown and MCL1 inhibition (Fig. 3i; Supplementary Fig. 3d).

To determine the effects of BCL2 family inhibition in vivo, we evaluated the efficacy of BCL2 inhibitor venetoclax and MCL1 inhibitor S63845 in an Myc-driven SCLC mouse model (RPM) ${ }^{6}$. As expected, BCL2 inhibition had no beneficial effect on overall survival of RPM mice (Supplementary Fig. 3e). While single agent S63845 (25 or $40 \mathrm{mg} / \mathrm{kg})$ and combined S63845/chemotherapy at $25 \mathrm{mg} / \mathrm{kg}$ of the MCL1 inhibitor had a modest effect on the survival of RPM mice, the $40 \mathrm{mg} / \mathrm{kg} \mathrm{S63845}$ and chemotherapy combination failed to improve survival of the mice beyond vehicle treatment (Supplementary Fig. 3e). While the trend for the higher efficacy of single agent MCL1 vs. BCL2 inhibition is consistent with our in vitro results, the limited affinity of $\mathbf{S 6 3 8 4 5}$ for murine MCL1 ${ }^{22,27}$ may mask otherwise stronger effects in this murine GEMM. In addition, S63845/chemotherapy regimens induced pronounced weight loss indicating high toxicity for the a

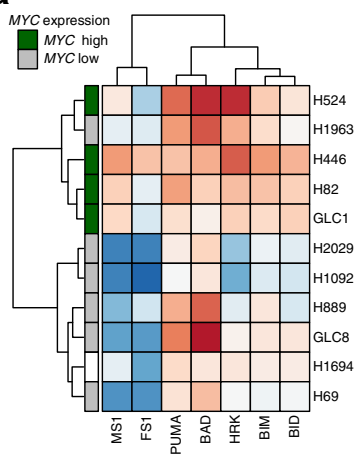

b

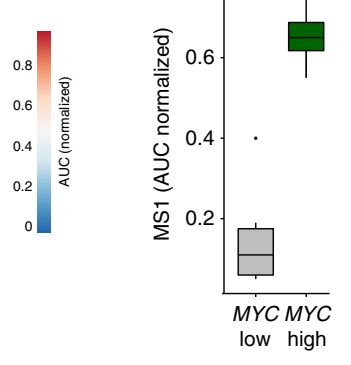

C

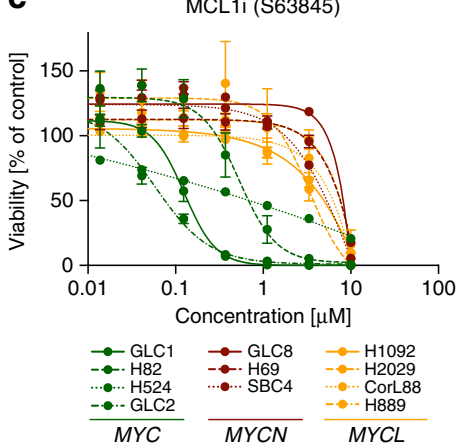

d

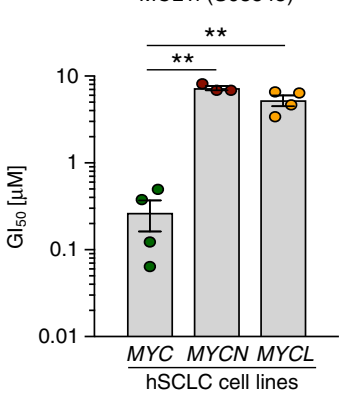

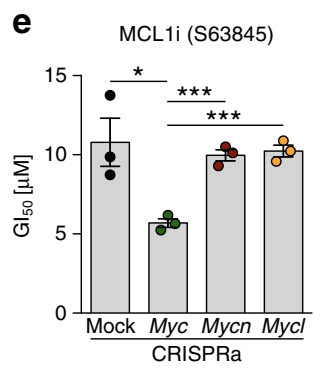

f

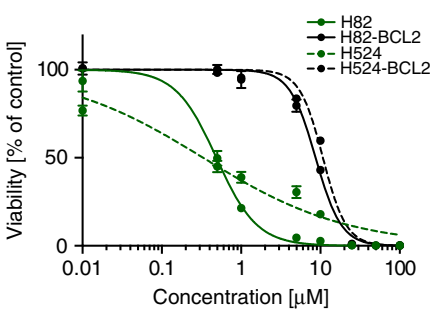

g

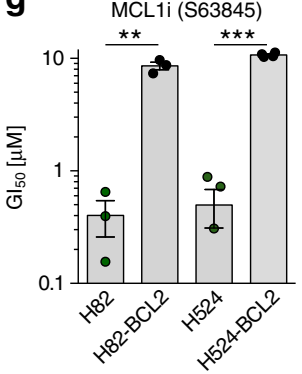

h

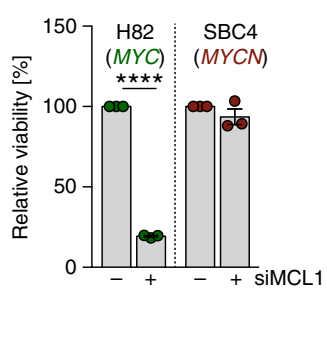

i

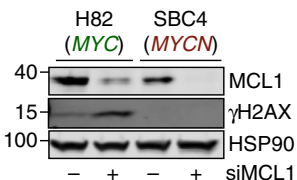

Fig. 3 MYC drives apoptotic priming and MCL1 dependency. a Heatmap of BH3 profiling showing sensitivity of human small cell lung cancer (SCLC) cell lines against specific apoptosis-inducing peptides. MYC expression in the individual cell lines is annotated on the left. $\mathbf{b}$ Sensitivity of SCLC cell lines toward apoptosis induction by sensitizer peptide MS1 as marker for MCL1-dependent apoptosis. Cell lines are grouped into MYC low and high expression. Sensitivity is calculated as area under the curve. Center line (median), lower/upper box hinges (25th/75th percentile), whiskers extend to the most extreme value within $1.5 \times$ interquartile range (IQR) of the hinges. c Cell viability screening of MYC ( $n=4 ; \mathrm{GLC1}, \mathrm{H} 82, \mathrm{H} 524, \mathrm{GLC2}), \mathrm{MYCN}(n=3 ; \mathrm{GLC} 8$,

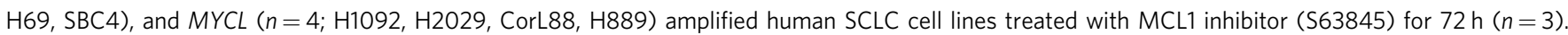
d $\mathrm{GI}_{50}$ values SCLC cell lines treated with $\mathrm{S63845}$. Cell lines are grouped according to their MYC status $(n=3)$. e $\mathrm{GI}_{50}$ values of $M y c$ paralog-activated CRISPRa cells treated with S63845. $\mathbf{f}$ Cell viability screening of MYC-amplified H82 and H524 cells $\pm B C L 2$ overexpression treated with S63845 ( $n=3$ ) $\mathbf{g ~ G l}{ }_{50}$ values of cell viability screening in $\mathbf{f}(n=3)$. h Relative cell viability of H82 (MYC-amplified) and H69 (MYCN-amplified) human SCLC cell lines $48 \mathrm{~h}$ after transfection with non-targeted small interfering RNA (siRNA) or siRNA directed against MCL1 $(n=3)$. i Western blot showing MCL1 and $\gamma \mathrm{H} 2 \mathrm{AX}$ levels in H82 (MYC-amplified) and H69 (MYCN-amplified) human SCLC cell lines $48 \mathrm{~h}$ after Ctrl. or MCL1 siRNA transfection. HSP90 was used as a loading control. Error bars indicate mean \pm SEM. Two-tailed unpaired $t$ tests, ${ }^{\star \star \star \star} p<0.0001,{ }^{\star \star \star} p<0.001,{ }^{\star \star} p<0.01,{ }^{\star} p<0.05$. Source data are provided as a Source Data file 
combination that prohibited a dose escalation for the MCL1 inhibitor (Supplementary Fig. 3f). Taken together, lack of BCL2 expression favors a potentially druggable MCL1 dependency in MYC-activated SCLC.

MYC triggers a druggable DDR in vivo. Next, we investigated the impact of cell cycle checkpoint inhibition on the induction of DDR and apoptosis. We observed that alisertib as well as volasertib treatment led to a rapid induction of $\gamma \mathrm{H} 2 \mathrm{AX}$ and CC3 levels in human $M Y C$-amplified and murine CRISPRa $M y c$-activated cells (Fig. 4a, b and Supplementary Fig. 4a, b). Using immunofluorescence, we observed increased steady-state levels of $\gamma \mathrm{H} 2 \mathrm{AX}$ $(p<0.0001$, one-way analysis of variance (ANOVA) $)$ and DNA double-strand break (DSB) marker 53BP1 $(p<0.0001$, one-way ANOVA) in $M y c$-activated murine SCLC cells suggesting DSBmediated activation of the DDR in these cells (Fig. 4c, d). Basal DDR activation was also observed in MYC-overexpressing human SCLC cells (Supplementary Fig. 4c, d). Further elevation of DNA damage by chemotherapeutics (etoposide and cisplatin) or checkpoint kinase 1 (CHK1) inhibitors (prexasertib, PF-477736, MK$8776)^{6,9}$ decreased viability preferentially in $M y c$-activated CRISPRa cells (Fig. 4e). Consistently, etoposide treatment of $M y c$-activated cells rapidly induced $\gamma \mathrm{H} 2 \mathrm{AX}$ and CC3 levels (Supplementary Fig. 4e). Interestingly, BCL2 overexpression reduced $\gamma \mathrm{H} 2 \mathrm{AX}$ levels after both etoposide and alisertib treatment of $M y c$-activated CRISPRa cells (Fig. 4f, g). We next combined AURK and CHK1 inhibition and observed synergistic activity at low nanomolar concentrations of alisertib and prexasertib in clonogenic and viability assays (Fig. 4h, i) with more pronounced synergy in $M y c$ activated cells (Supplementary Fig. 4f).

In SCLC patients who are routinely treated with first-line cisplatin/etoposide combination chemotherapy, emergence of chemo-resistance is rapid and frequent. To assess the efficacy of combined AURK/CHK1 inhibition in the setting of chemoresistance, we generated chemo-resistant cell lines from $M y c$ activated CRISPRa cells (Supplementary Fig. 4g) and subjected these cells to combined AURK/CHK1 inhibition. We observed that chemo-resistance substantially increased resistance against combined AURK/CHK1 inhibition (Supplementary Fig. 4h) arguing for efficacy of this treatment strategy in the first-line setting but not upon chemo-resistance. Interestingly, chemoresistant cells displayed increased AURK and CHK1 phosphorylation levels suggesting higher activity of both enzymes after chemo-resistance (Supplementary Fig. 4i).

We next sought to validate the efficacy of AURK/CHK1 inhibition in vivo in the $M y c$-driven RPM mouse model. Compared to vehicle treatment, CHK1 inhibitor treatment (prexasertib) prolonged survival of RPM mice similar to chemotherapy (cisplatin/etoposide) (Fig. 4j). The combination of prexasertib and AURK inhibitor (alisertib) further prolonged survival of RPM mice compared to chemotherapy (median survival 22.5 vs. 28 days; $p=0.005$, Log-rank (Mantel-Cox) test; Fig. 4j). Furthermore, the combination of either prexasertib or alisertib with chemotherapy was slightly less effective (median survival 19 and 26 days) than the alisertib/prexasertib combination (Fig. 4j). Vehicle-treated mice exhibited rapid tumor growth while chemotherapy- or prexasertib-treated animals exhibited a modest delay in tumor growth followed by rapid relapse (Fig. $4 \mathrm{k}$ and Supplementary Fig. 4j). Of importance, mice treated with the combination of targeted therapy agents, alisertib and prexasertib, exhibited moderate weight loss compared to regimens in which targeted agents were combined with chemotherapy (Supplementary Fig. 4k). This suggests manageable toxicity of combined AURK/CHK1 inhibition and further strengthens our model in which
MYC-dependent tumors are more susceptible to perturbation of the cell cycle and DDR control pathways (Fig. 4i).

\section{Discussion}

Here we investigated how MYC paralogs modulate drug dependencies in SCLC. We developed an isogenic CRISPRa-based model to study the endogenous activation of the different MYC paralogs in GEMM-derived SCLC cell lines. This cellular system allowed us to molecularly define and phenotypically characterize MYC-paralog-driven SCLC uncoupled from the divergent genetic background of patient-derived cell lines.

In summary, our data provide mechanistic insight into $M Y C$ paralog-specific dependencies with direct implications for a personalized treatment against SCLC tumors. Our findings reveal a pivotal role for BCL2 as a major regulator of response to cell cycle and DNA damage checkpoint inhibitors. In SCLC cells, MYC activation represses $B C L 2$ thereby limiting the pool of antiapoptotic proteins. Indeed, we observed increased apoptotic priming and a strong MCL1 dependency in MYC-overexpressing cells, which are also vulnerable to direct and indirect DNA damage induction (Fig. 4l). Differential MYC protein levels do not alter MCL1 expression, so lack of BCL2 likely is the main driver of MYC-induced MCL1 dependency. Consistent with previous reports ${ }^{29}$, reintroduction of BCL2 mitigated DNA damage-induced cell death. The specific silencing of an antiapoptotic protein such as BCL2 seems to be paradoxical since the benefit to MYC-activated cells is not obvious. A study by Ichim and colleagues described limited mitochondrial outer membrane permeabilization (MOMP), a phenomenon termed minority MOMP, as a trigger for cellular transformation and tumorigenesis $^{30}$. In this scenario, limited caspase activity promotes DNA damage induction and genome instability. The steady-state $\gamma \mathrm{H} 2 \mathrm{AX}$ levels in cells with high MYC strongly resemble such a limited MOMP baseline. Following this hypothesis, suppression of BCL2 may facilitate the induction of this phenotype. On the other hand, increased levels of $\gamma \mathrm{H} 2 \mathrm{AX}$ may contribute to DNA damage accumulation in MYC-activated cells following chemotherapy and/or CHK1 inhibition. Furthermore, a disruption of the G2/M checkpoint via AURK inhibition, in the background of TP53/RB1-loss-induced defective G1/S checkpoint, may have the same cytotoxic effects. Failure to repair accumulated DNA lesions likely induces apoptosis followed by cell death (Fig. 4l).

We show that this MYC-specific vulnerability can be therapeutically exploited in vitro and in vivo by combined AURK/ CHK1 inhibition. A translation of this regimen into a clinical setting might primarily be effective in a first-line setting since chemo-resistant cell lines were also resistant to combined AURK/ CHK1 inhibition. The combination of two targeted therapy agents at tolerable doses might overcome the need for pan-toxic chemotherapy. Since the efficacy of combining targeted therapy with chemotherapy was also superior compared to chemotherapy alone, this strategy might prevent or delay the emergence of resistance. We observed less toxicity for combined AURK/CHK1 inhibition, which might overcome previously observed hematological toxicities for alisertib, especially in combination with chemotherapy ${ }^{31}$. Several clinical trials are already evaluating AURK (NCT03216343, NCT03092934, NCT02719691, NCT02134067, NCT01118611) and CHK1 (NCT02735980, NCT02797964, NCT02797977, NCT02873975) inhibitors either as single agents or in combination with chemotherapy in SCLC patients. A recently completed Phase II trial (NCT02038647) that investigated the effects of alisertib in combination with paclitaxel as second-line therapy for SCLC initially reported a significant increase in progression-free survival ${ }^{32}$. Interestingly, retrospective analysis of a subset of 
a

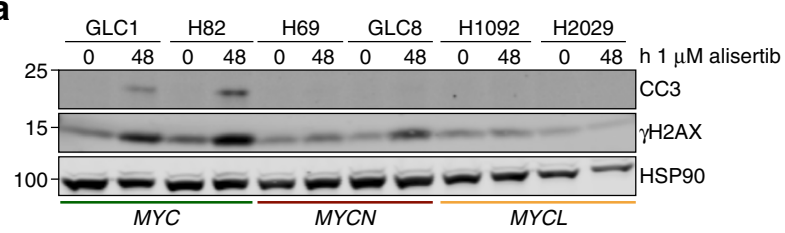

C

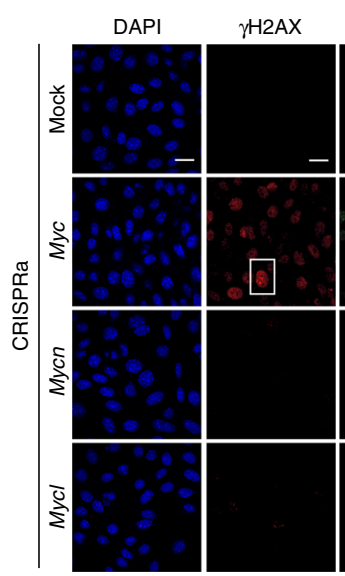

f

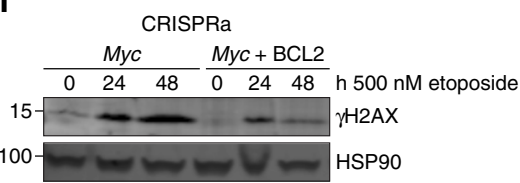

i

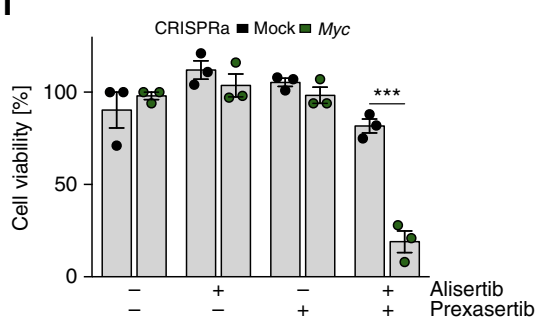

$\mathbf{k}$

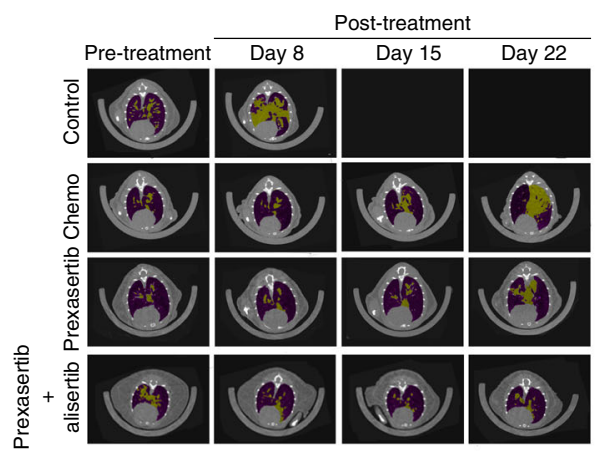

j

I b
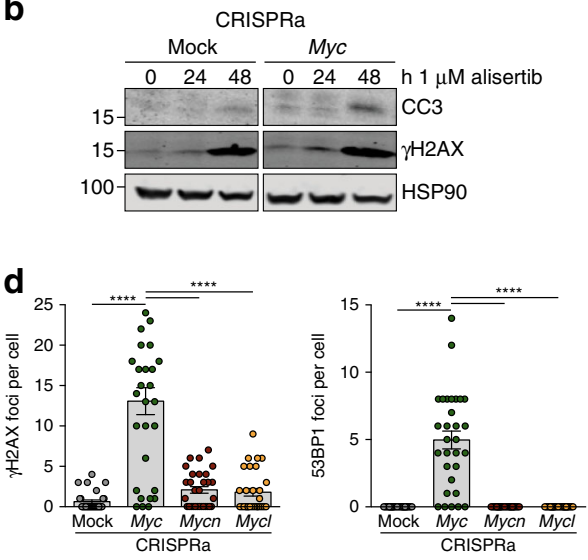

e

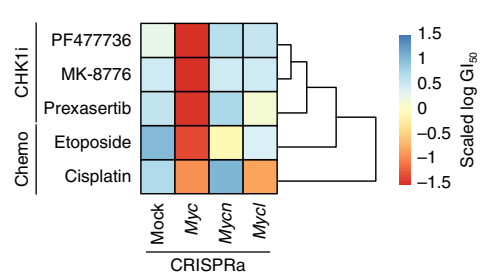

g

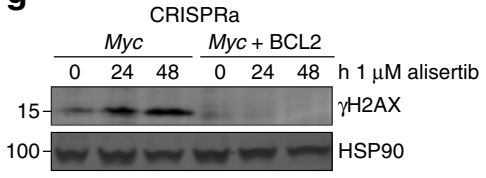

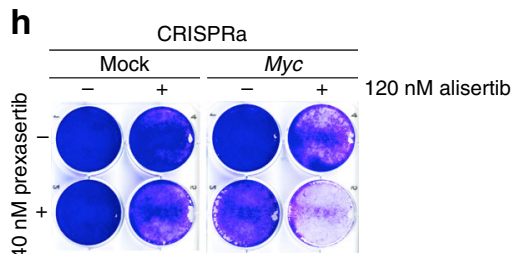

h
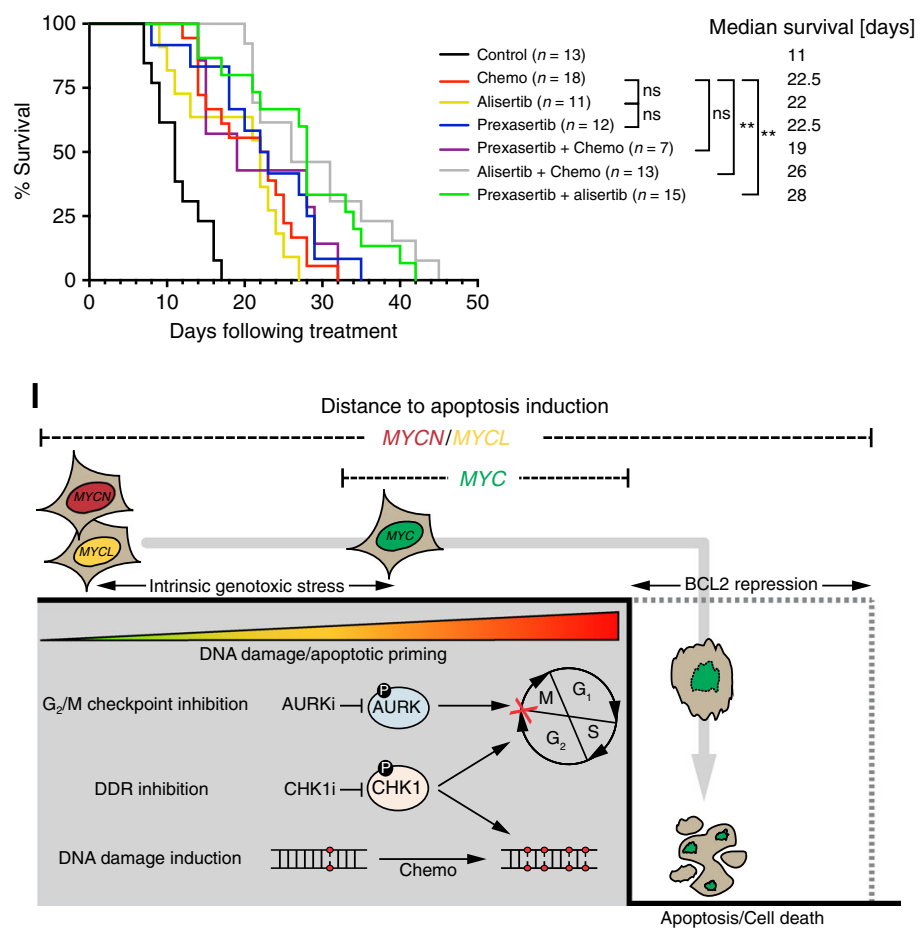

patients revealed that the alisertib/paclitaxel combination preferentially improved survival of patients with high MYC protein expression $^{33}$. This is in line with our data that suggest high MYC expression is predictive of response to AURK and/or CHK1 inhibition. Therefore, our data may facilitate the selection of patients who particularly benefit from this treatment, reducing unnecessary toxicities. Thus our study bolsters the mechanistic understanding of the role of specific MYC paralogs for the fine-tuning of the apoptotic machinery and druggable dependencies in SCLC. 
Fig. 4 MYC triggers a druggable DNA-damage response (DDR) in vivo. a, b Western blot of cleaved caspase 3 (CC3) and $\gamma \mathrm{H} 2 \mathrm{AX}$ in MYC-variant-amplified human small cell lung cancer (SCLC) cell lines $(n=6)(\mathbf{a})$ or Myc-activated CRISPRa cells $(\mathbf{b})$ treated with alisertib for the indicated times. HSP90 was used as a loading control. c Representative images of immunofluorescence (IF) experiments of Myc paralog-activated CRISPRa cells showing DAPI (DNA), $\gamma \mathrm{H} 2 \mathrm{AX}$ (DDR activation), and 53BP1 (DNA double-strand breaks) staining (Scale bar: $20 \mu \mathrm{m}$ ). $\mathbf{d}$ Quantification of $\mathbf{c}$ showing mean number of $\gamma \mathrm{H} 2 \mathrm{AX}$ (top) and 53BP1 (bottom) foci per cell $(n=30)$. Error bars indicate mean \pm SEM. One-way analysis of variance, ${ }^{\star \star \star \star} p<0.0001$. e Heatmap displaying sensitivity (scaled $\log \left(\mathrm{Gl}_{50}\right)$ ) of Myc paralog-activated CRISPRa cells treated with CHK1 inhibitors (MK8776, PF477736, prexasertib) or chemotherapeutics (etoposide, cisplatin) for $96 \mathrm{~h}(n=3)$. f, $\mathbf{g}$ Western blot of $\gamma \mathrm{H} 2 \mathrm{AX}$ in Myc-activated CRISPRa cells \pm BCL2 overexpression treated with etoposide (g) and alisertib (h). HSP90 was used as a loading control. $\mathbf{h}$ Crystal violet assay of control and Myc-activated CRISPRa cells upon treatment with $120 \mathrm{nM}$ alisertib, $40 \mathrm{nM}$ prexasertib, and combined treatment for $96 \mathrm{~h}$. $\mathbf{i}$ Viability of mock control and Myc-activated CRISPRa cells upon treatment with $120 \mathrm{nM}$ alisertib, $40 \mathrm{nM}$ prexasertib, and combined treatment for $96 \mathrm{~h}(n=3)$. Error bars indicate mean \pm SEM. Two-tailed unpaired $t$ tests, ${ }^{\star \star \star} p<0.001$. $\mathbf{j}$ Survival analysis of RPM mice bearing MYC-driven SCLC treated with vehicle control (phosphate-buffered saline (PBS), $n=13$ ), chemotherapy (cisplatin/etoposide, $n=$ 18), Aurora Kinase (AURK) inhibitor alisertib $(n=11)$, checkpoint kinase 1 (CHK1) inhibitor prexasertib $(n=12)$, prexasertib+chemotherapy $(n=7)$, alisertib+chemotherapy $(n=13)$, and prexasertib+alisertib $(n=15)$. Log-rank (Mantel-Cox) test, ${ }^{\star *} p<0.009$. k Representative micro-computed tomographic images of RPM mice pre-treatment and after treatment with vehicle control (PBS), chemotherapy (cisplatin/etoposide), CHK1 inhibitor prexasertib, and prexasertib combined with AURK inhibitor alisertib. Tumors are colored in yellow, air space in purple. I Model of MYC paralog-dependent apoptotic priming and vulnerabilities in SCLC. Source data are provided as a Source Data file

\begin{abstract}
Methods
Cell culture. Human SCLC cell lines were obtained from ATCC and verified by STR profiling at the University of Utah DNA sequencing core facility or at the Institute for Forensic Medicine of the University Hospital of Cologne. GLC1, GLC2, H82, H524, GLC8, SBC4, H69, COR-L303, SBC7, COR-L88, MEF, and Myc-activated CRISPRa cells were cultured in RPMI; H1092, H2029, and H889 were cultured in HITES; NIH3T3 and HEK293T cells were cultured in DMEM. All media were supplemented with $10 \%$ fetal bovine serum, $1 \%$ Penicillin/Streptomycin, and $1 \% \mathrm{~L}$-glutamine. All cells were grown at $37^{\circ} \mathrm{C}$ in a humidified atmosphere with $5 \% \mathrm{CO}_{2}$.
\end{abstract}

Reagents. For cell culture studies, drugs were dissolved in dimethyl sulfoxide (DMSO) to a final stock concentration of $10 \mathrm{mM}$ except for prexasertib $(4.5 \mathrm{mM})$. With the exception of prexasertib (MedChemExpress), all compounds were purchased from Selleckchem.

CRISPR activation. Briefly, sgRNA sequences (see Supplementary Table 2) targeting promoters of $M y c, M y c n$, and $M y c l$ were obtained from the sgRNA design tool (http://sam.genome-engineering.org/database/, Cas9-Activators with SAM, accessed 12/2015) and cloned into lentiSAMv2. Lentiviral particles of lenti-MS2p65-HSF1_Hygro and lentiSAMv2 (containing $M y c$ paralog sgRNAs) were produced in HEK293T cells co-transfected with pMD.2 and pCMVd.8.9 helper plasmids. Target cells were first transduced with lentiviral particles of lentiMS2-p65HSF1_Hygro followed by hygromycin selection $(400 \mu \mathrm{g} / \mathrm{ml})$. Selected cells were then transduced with lentiviral particles of lentiSAMv2 followed by blasticidin selection $(1.5 \mu \mathrm{g} / \mathrm{ml})$.

Cell viability screening. To assess cell viability, cells were plated in 96 -well plates in triplicates and compounds were added at 8 decreasing compound concentrations $24 \mathrm{~h}$ after seeding. Seventy-two hours later, cell viability was measured via Cell Titer-Glo (CTG) assay (Promega) and was normalized to DMSO-treated controls. Half-maximal growth inhibitory $\left(\mathrm{GI}_{50}\right)$ concentrations of cell viability were inferred by fitting sigmoidal dose-response curves using the Prism 8 software (GraphPad). Data are represented as mean \pm SEM and significance was calculated by unpaired Student's $t$ tests.

Cell proliferation kinetics. In all, $2 \times 10^{4}$ cells were plated in triplicate in one well of a 12-well plate. Cell number was determined daily for 4 consecutive days. Data are presented as mean \pm SEM.

Whole-exome sequencing. DNA from $M y c$-paralog-activated cells was extracted using the Gentra Puregene Tissue Kit (Qiagen) according to the manufacturer's instructions. Library preparation for exome sequencing was performed with the SureSelectXT Library Prep Kit and the Target Enrichment Kit using the Mouse All Exon Capture ab (Agilent, USA) following the SureSelectXT Automated Target Enrichment Illumina PE Multiplexed Seq protocol. Sequencing was performed with a $2 \times 76$ bp protocol on a HiSeq4000. Raw sequencing reads were aligned to the mouse reference genome mm10 using BWA-MEM, followed by trimming of overlapping read pairs, and removal of PCR duplicates and secondary alignments. For copy number $(\mathrm{CN})$ analysis, Sclust $^{34}$ is applied to estimate purity-corrected CNs by conditionally optimizing likelihoods of allelic imbalances and read ratios relative to available mouse normal data. All sequencing data will be released upon publication. Sequencing data are deposited at EBI Array Express, accession \# E-MTAB-7412.
Transcriptome data analysis. Human SCLC RNA-seq cell line generated within this study and SCLC cell line raw data used previously ${ }^{6}$ were aligned to the human reference genome $\mathrm{Hg} 38$ using $\mathrm{STAR}^{35}$ followed by gene expression quantification as transcript per million (TPM) and counts using RSEM ${ }^{36}$. For differential gene expression, cell lines were grouped according to MYC expression into MYC-high ( $n=22$, COR.L279, CPC.N, DMS114, DMS273, DMS454, DMS53, GLC1, GLC2, H1048, H1341, H1930, H2171, H446, H524, H82, H841, NCI.H146, NCI.H2081, NCI.N417, SCLC.21 H, SHP77, SW1271) and MYC-low $(n=20$, COR.L303,COR L47, COR.L88, DMS153, DMS79, GLC8, H1836, H196, H1963, H2029, H209C, H2141, H526, H69, H889, NCI.H1092, NCI.H187, NCI.H1882, NCI.H345, SBC7). Differential gene expression between groups was calculated from count-level data using DESeq2 ${ }^{37}$. Resulting $p$ values were adjusted using Benjamini-Hochberg correction. Annotation of MYC paralog amplification status in human SCLC cell lines was obtained from published genomic data ${ }^{7,20,38}$. 3' RNA-seq data was aligned to the mouse reference genome GRCm38 using STAR and quantified with RSEM prior to downstream analysis. Processed human primary SCLC tumor sample data were acquired from a published study ${ }^{1}$. Primary samples were classified as MYC family member high vs. low based on gene expression, where cutoffs were derived from Gaussian-mixture models. In brief, samples were grouped by fitting two normal distributions to log-transformed expression of the MYC family member. Cut-offs between high and low expression groups were derived using the respective fitted distributions. Publicly available RNAseq data for a cohort of 79 SCLC patients ${ }^{15}$ was obtained from GEO (GSE60052) including normalized $\log _{2}$-transformed expression per gene. Patients were categorized in 15 bins based on MYC expression. Median expression levels of MYC and BCL2 per bin were calculated and correlated using Spearman correlation coefficient. To assess RNA expression of $\mathrm{Bcl} 2$ in mouse tumor models, we used published expression data including RNAseq of RPM $(n=11)$ and RPR2 $(n=4)$ mouse models ${ }^{6}$, supplemented with gene expression array data for (RP $(n=10)$ and RPP130 $(n=3)$ mouse tumors (GSE18534) ${ }^{39}$. $\log _{2}$-transformed intensity values were averaged per gene if multiple probes were present. To account for potential effects of expression analysis method, $\log _{2}$-FPKM values and $\log _{2}$-intensity values were transformed to $z$-scores per sample followed by quantile normalization per gene across samples prior to joined analysis. To assess correlation of MYC and BCL2 mRNA expression across various cancer entities, cell line RNAseq data generated by the CCLE was downloaded from www.depmap.org (Release 19Q1). To account for entity-specific baseline expression differences of MYC and BCL2, $\log _{2}$-transformed expression levels quantified as TPM were first scaled per gene within each of the 27 tumor entities before calculating Pearson correlation.

Cell cycle analysis by flow cytometry. A total of $5 \times 10^{5}$ cells were seeded in 6 well plates and incubated overnight, before addition of $2 \mathrm{mM}$ thymidine for $16 \mathrm{~h}$ (first block). After the first block, cells were washed twice with phosphate-buffered saline (PBS) and incubated in growth medium for $8 \mathrm{~h}$ before addition of $2 \mathrm{mM}$ thymidine for $16 \mathrm{~h}$ (second block). Cells were washed twice with PBS and released. Every $2 \mathrm{~h}$ in a period of $12 \mathrm{~h}$, cells were trypsinized, washed with PBS, fixed with $70 \%$ ethanol, and incubated for half an hour on ice. Fixed cells were stored at $4{ }^{\circ} \mathrm{C}$ for the cell cycle analysis. Ethanol-fixed cells were centrifuged for 5 min at $300 \times g$, washed twice with cold PBS, and centrifuged for $5 \mathrm{~min}$ at $300 \times g$. Cells were then incubated with $100 \mathrm{mg} / \mathrm{ml}$ DNase-free RNaseA in PBS for $30 \mathrm{~min}$ on ice. Next, cells were washed with PBS and incubated with $100 \mathrm{mg} / \mathrm{ml}$ propidium iodide (PI) for 30 min at room temperature (RT) in the dark. Finally, cells were analyzed in a flow cytometer (BD Biosciences). PI fluorescence was determined using FL-3 channel, $488 \mathrm{~nm}$. Raw data were analyzed with the FlowJo software.

MIZ1/MYC co-immunoprecipitation. HEK293T cells were transfected with pcDNA-HA-HA-MYC, pcDNA-HA-MYCN or pcDNA-HA-HA-MYCL in 
combination with pcDNA-MIZ1. Two days post-transfection, cells were harvested and subjected to MIZ1 IP using anti-MIZ1 antibody (sc-139685, Santa Cruz Biotechnology, $4 \mu \mathrm{g}$ ). Antibody-protein complexes were captured using $20 \mu \mathrm{l}$ protein $\mathrm{G}$ sepharose beads (Santa Cruz Biotechnology). Immunoprecipitates were then analyzed by western blot.

GDSC methylation data analysis. Publicly available human SCLC cancer cell line data ${ }^{20}$ including gene expression were obtained from http://www. cancerrxgene.org/ (Genomics of Drug Sensitivity in Cancer Project) and corresponding Illumina 450k methylation beta values (GSE68379) were downloaded from www.ncbi.nlm.nih.gov/geo (NCBI Gene Expression Omnibus, both accessed 27 Dec 2017). SCLC cell lines were classified as MYC high vs. MYC low based on RMA normalized basal MYC expression levels as described above. For methylation analyses, CpGs were filtered using a detection $p$ value $<0.01$ followed by removal of probes containing single-nucleotide polymorphisms, nonCpG probes, and cross-reactive probes ${ }^{40}$. Prior to further downstream analysis, beta values were normalized by peak-based correction ${ }^{41}$. Illumina $450 \mathrm{k}$ array annotation files were used to select probes in the BCL2 gene body and promoter region.

Drug combination screening. Cells were plated in a $6 \times 6$ matrix of wells of a 96 well plate and treated with alisertib and prexasertib in various independent concentration pairs (concentrations were fixed ranging from $40 \mathrm{nM}$ to $3.3 \mu \mathrm{M}$ for alisertib and from $10 \mathrm{nM}$ to $1.1 \mu \mathrm{M}$ for prexasertib) for $96 \mathrm{~h}$ followed by viability measurement using CTG assay. Results of three replicate experiments were pooled and synergy was calculated applying a Bliss independence model using the $\mathrm{R}$ package synergyfinder 42

Cycloheximide shutoff experiments. Cells were seeded and pre-treated with DMSO (control) or $1 \mu \mathrm{M}$ alisertib for $24 \mathrm{~h}$ before addition of $100 \mu \mathrm{g} / \mathrm{ml}$ cycloheximide for $0,1,2,3$, and $4 \mathrm{~h}$. Cell lysates were prepared and analyzed by western blot. Protein amounts of MCL1 were calculated by the Image Studio Software (LICOR Biosciences) and normalized to HSP90 amounts.

shRNA knockdown experiments. shRNA targeting $M y c$ (TGTAAGCTTCAGCC ATAATTT) was cloned into a Tet-pLKO-puro vector and cotransfected with pMD2.G and pCMVd.8.9 helper plasmids into HEK 293T cells using TransIT-LT1 reagent (Mirus). Forty-eight hours post-transfection, replication-incompetent lentiviruses were collected from the supernatant for infection in the presence of 8 $\mu \mathrm{g} / \mathrm{ml}$ polybrene. Twenty-four hours after infection, growth medium was changed and $3 \mu \mathrm{g} / \mathrm{ml}$ puromycin was added for selection. After 5 passages, $M y c$ knockdown was induced by addition of doxycycline $(500 \mathrm{ng} / \mathrm{ml})$ and $M y c$ knockdown confirmed by RT-qPCR and immunoblot. For compound screenings, doxycycline was added when cells were plated.

siRNA knockdown experiments. siRNA pools targeting MCL1 (siMCL1\#1 GGUUUGGCAUAUCUAAUAA, siMCL1\#2 GAAGGUGGCAUCAGGAAUG, siMCL1\#3 GAUUAUCUCUCGGUACCUU, siMCL1\#4 CGAAGGAAGUAUCGA AUUU), or DNMT3a (siDNMT3A\#1 GCAUUCAGGUGGACCGCUA, siDNMT3 A\#2 GCACUGAAAUGGAAAGGGU, siDNMT3A\#3 CUCAGGCGCCUCAGAG CUA, siDNMT3A\#4 GGGACUUGGAGAAGCGGAGS) were purchased from Dharmacon and transfected at $20 \mathrm{nM}$ final concentration into SCLC cell lines (H82, SBC4, GLC1, GLC2) using Dharmafect Transfection Reagent \#2 (Dharmacon). Growth medium was changed after $12 \mathrm{~h}$. Experiments assessing knockdown efficiency, cell viability, gene expression, and immunoblots to determine knockdown effects were performed $48 \mathrm{~h}$ post-transfection.

Protein overexpression experiments. Vectors pMSCV-PIG (puro-IRES-GFP) and pMSCV-PIG-BCL2 were cotransfected with pMD.2 and pCMVd.8.9 helper plasmids into HEK 293T cells using TransIT-LT1 reagent (Mirus), respectively. Forty-eight hours post-transfection, replication-incompetent lentiviruses were collected from the supernatant for infection of Myc CRISPRa cells and H82 and $\mathrm{H} 524$ cells in the presence of $8 \mu \mathrm{g} / \mathrm{ml}$ polybrene. Twenty-four hours after infection, growth medium was changed and $3 \mu \mathrm{g} / \mathrm{ml}$ (Myc CRISPRa cells) or $1 \mu \mathrm{g} / \mathrm{ml}$ (H82/ H524) puromycin was added for selection for the duration of 6 days (3 passages). After selection, cells were analyzed for protein expression.

RNA isolation qRT-PCR. Total RNA was isolated using the Qiazol reagent (Qiagen) according to the manufacturer's instructions. In all, $1.5 \mu \mathrm{g}$ of total RNA was subjected to DNaseI (Sigma) digestion and reverse transcribed using SuperscriptIII (Thermo Fisher Scientific) with random hexamer primers. Quantitative real-time PCR (qPCR) was performed using 7900HT Real-Time PCR System (Applied Biosystems) and the Power SYBR Green PCR Master Mix (Thermo Fisher Scientific). The qPCR primers used to analyze mRNA levels are listed in Supplementary Table 2. Data were normalized to $18 \mathrm{~S}$ rRNA levels and are presented as mean \pm SEM and significance was calculated by unpaired Student's $t$ tests.
RNA sequencing. Total RNA was isolated using the RNeasy Mini Prep Kit (Qiagen) according to the manufacturer's instructions with a 75-bp paired-end protocol on a HiSeq4000 (Illumina, USA). 3' UTR RNA sequencing libraries for murine CRISPRa cells were prepared using the QuantSeq 3' mRNA-Seq Library Kit (Lexogen, Austria) and sequenced with a 50-bp single-end protocol on an Illumina HiSeq4000 (Illumina, USA). Sequencing data are deposited at EBI Array Express, accession \# E-MTAB-7411.

Chromatin immunoprecipitation. Cells were crosslinked in $1 \%$ formaldehyde, and chromatin was extracted and sonicated. Equal amounts of chromatin were incubated overnight with specific antibodies against MYC (clone 9E11, ab56, Abcam, $5 \mu \mathrm{g}$ ), MYCN (clone B8.4.B, sc-53993, Santa Cruz Biotechnology, $4 \mu \mathrm{g}$ ), DNMT3a (ab2850, Abcam, $4 \mu \mathrm{g}$ ), MIZ1 (clone 10E2, Elmar Wolf, Würzburg, $15 \mu \mathrm{l}$ antiserum), or unspecific mouse IgG (sc-2025, Santa Cruz Biotechnology, $4 \mu \mathrm{g}$ ). ChIP complexes were captured using protein G Dynabeads (Thermo Fisher Scientific), washed, eluted, and decrosslinked. DNA was purified using the ChIP DNA Clean \& Concentrator Kit (Zymo Research) and analyzed by qRT-PCR using primers listed in Supplementary Table 2. ChIP signals of non-specific background (IgG) were subtracted from specific antibody ChIP signals. ChIP signals were calculated as percentage of input. Data are presented as mean \pm SEM and significance was calculated by unpaired Student's $t$ tests.

BH3 profiling assay. Cells were pelleted, washed in PBS, resuspended in MEB2 buffer, and $1 \times 10^{4}$ to $2 \times 10^{4}$ cells were added to each well of a 384 non-binding plate containing MEB $2+20 \mu \mathrm{g} / \mathrm{ml}$ digitonin + sensitizer peptides at $2 \times$ the final concentration. Permeabilized cells were incubated for $1 \mathrm{~h}$ at RT in the presence of peptides, fixed by the addition of formaldehyde to $1 \%$ final concentration for $10 \mathrm{~min}$ at RT, and neutralized by the addition of N2 buffer (Tris/glycine) to terminate fixation. Cells were stained overnight by adding Alexa647-Cytochrome C (clone 6H2. B4, Biolegend) to $250 \mathrm{ng} / \mathrm{ml}$ final concentration and Hoechst 33342 to $1 \mu \mathrm{g} / \mathrm{ml}$ final concentration. Analysis was conducted on a BD Fortessa or BD Fortessa X20 with gating on DAPI+ singlets and normalization of the Cytochrome $\mathrm{C}$ mean fluorescent intensity values to the buffer alone and $25 \mu \mathrm{M}$ alamethicin controls.

Immunofluorescence. Murine $M y c$ paralog-activated cells were grown on glass coverslips and human SCLC cells were grown on NuncTM Lab-TekTM coated with Gelatine solution $0.1 \%$ in PBS (PAN Biotech). Cells were fixed with $4 \%$ paraformaldehyde at RT, permeabilized in PBS containing $0.25 \%$ Triton X-100, and blocked in PBS containing $0.2 \%$ Tween 20 and 3\% bovine serum albumin Cells were incubated overnight with primary antibodies to $\gamma \mathrm{H} 2 \mathrm{AX}$ (\#05-636, Merck, 1:500), MCL-1 (sc-819, Santa Cruz Biotechnology, 1:100), or 53BP1 (MAB3802, Merck, 1:500). After washing, cells were incubated with secondary antibodies conjugated to Alexa Fluor-488 (A11029, Thermo Fisher Scientific, 1:1000) and Alexa Fluor-647 (A32733, Thermo Fisher Scientific, 1:1000) in combination with DAPI (4',6-diamidino-2-phenylindole; Sigma, 1:1000). Coverslips were mounted using Fluromount-GTM (Thermo Fisher Scientific). Microscopy was performed using a Zeiss Meta 710 confocal microscope and images were analyzed by the ImageJ software.

Bisulfite sequencing. Cellular DNA was extracted using the Puregene Kit (Qiagen) according to the manufacturer's instructions. Five hundred nanograms of DNA were bisulfite converted using the EZ DNA Methylation-Gold Kit (Zymo Research) according to the manufacturer's instructions. Bisulfite-converted DNA was subjected to methylation-specific PCR using specific primers for the BCL2 promoter listed in Supplementary Table 2. PCR product was resolved on a $2 \%$ agarose gel and purified using the Monarch DNA Gel Extraction Kit (New England BioLabs), cloned into pCR4-TOPO TA Vector (Thermo Fisher Scientific), transformed into XL10-Gold Ultracompetent Cells (Agilent Technologies), and plated onto ampicillin selection LB-agar plates. DNA of single colonies was extracted using the NucleoSpin Plasmid EasyPure Mini Kit (Macherey-Nagel) and submitted to Sanger sequencing using sequencing primers M13-for and M13-rev (see Supplementary Table 2). Obtained sequences were analyzed and DNA methylation plots were generated using the QUMA quantification tool for methylation analysis ${ }^{43}$.

Crystal violet assay. In all, $2 \times 10^{5}$ cells were plated into one well of a 6-well plate and treated with DMSO (control), $40 \mathrm{nM}$ prexasertib, $120 \mathrm{nM}$ alisertib, and the combination of prexasertib and alisertib. Seventy-four hours after treatment, cells were fixed in $4 \%$ paraformaldehyde in PBS, stained with $0.1 \%$ crystal violet in PBS, and rinsed in PBS before image acquisition.

Generation of chemo-resistant cells. $M y c$-activated CRISPRa cells were subjected to prolonged etoposide treatment at increasing concentrations starting from $500 \mathrm{nM}$ for several weeks. The resulting, proliferating cell line was maintained in growth medium containing $2 \mu \mathrm{M}$ etoposide.

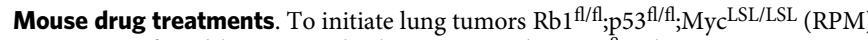
mice were infected by intratracheal injection with $1 \times 10^{8}$ Ad-CGRP-Cre virus 
(University of Iowa Virus Vector Core). Mice were imaged with a Quantum FX or GX2 microCT system (Perkin Elmer) and randomized into treatment groups upon detection of $\sim 10 \%$ lung tumor burden. Treatment groups included PBS control ( $n=15)$, chemotherapy (cisplatin/etoposide, $n=18)$, prexasertib $(n=12)$, or prexasertib combined with alisertib $(n=15)$. Prexasertib $(10 \mathrm{mg} / \mathrm{kg}$ in Captisol) was administered via subcutaneous flank injection twice a day on a weekly schedule of 2 days on and 5 days off. Prexasertib was provided by Dr. Lauren Byers and manufactured by the Institute for Applied Chemical Science at MD Anderson, Houston, TX. Alisertib (Apexbio Technology; $20 \mathrm{mg} / \mathrm{kg}$ in $10 \% \beta$-cyclodextrin) was administered via oral gavage twice a day on a weekly schedule of 5 days on and 2 days off. For weekly chemotherapy treatments, cisplatin (Sigma-Aldrich; $5 \mathrm{mg} / \mathrm{kg}$ in PBS) was administered on day 1 and etoposide (Sigma-Aldrich; $10 \mathrm{mg} / \mathrm{kg}$ in $70 \%$ PEG in water) was given on day 2 by intraperitoneal injection. To decrease toxicity, mice treated with prexasertib and chemotherapy received cisplatin on day 1, etoposide on day 2 , and prexasertib on days 5 and 6 of each weekly cycle. After 4 cycles of cisplatin/etoposide chemotherapy, mice were treated weekly with etoposide only. MCL1 inhibitor S63845 (25 mg/kg or $40 \mathrm{mg} / \mathrm{kg}$ in $20 \% \beta$-cyclodextrin with $25 \mathrm{mM} \mathrm{HCl}$ ) was administered by tail vein injection. Mice were treated with MCL1i at $25 \mathrm{mg} / \mathrm{kg}$ twice/week or $40 \mathrm{mg} / \mathrm{kg}$ once/week, and both treatment doses were tested in combination with cisplatin/etoposide chemotherapy. Since neither the $25 \mathrm{mg} / \mathrm{kg}$ nor $40 \mathrm{mg} / \mathrm{kg}$ monotherapy significantly improved survival, these groups were combined for data analysis. Both tested doses of S63845 + chemotherapy induced significant weight loss and toxicity. ABT- $199(50 \mathrm{mg} / \mathrm{kg}$ in $60 \%$ Phosal50, 30\% PEG400, 10\% ethanol) was administered by oral gavage once per day on a weekly schedule of 5 days on/2 days off. Mice were imaged at the start of each treatment cycle and 4 days post cisplatin, and images were quantified using the Analyze 11.0 (AnalyzeDirect) software. Endpoints for survival studies included labored breathing, $>20 \%$ weight loss, or signs of toxicity. Mice were sacrificed via $\mathrm{CO}_{2}$ asphyxiation prior to necropsy. Survival curve analysis was performed with the GraphPad Prism software. These experiments were approved by the HCI Institutional Animal Care and Use Committee (IACUC), and mice were housed in a specific pathogen-free barrier facility.

Micro-computed tomographic (microCT) imaging. Mice were scanned for $34 \mathrm{~s}$ under isoflurane anesthesia using a small animal Quantum FX or GX2 microCT (PerkinElmer) at $45 \mu \mathrm{m}$ resolution, $90 \mathrm{kV}$, with $160 \mathrm{~mA}$ current. Images were acquired using the PerkinElmer Quantum FX software and processed with Analyze 11.0 (AnalyzeDirect). Scans were calibrated for Hounsfield Units (HU) by determining the mean value of "Bed" and "Air" for representative scans using the region of interest (ROI) tool. Those values were matched to their known HU (40 and $-1000 \mathrm{HU}$, respectively) by the "Image Algebra" tool. A $3 \times 3 \times 3$ Median Filter was applied to every image using the "Spatial Filters" window. Thresholds for "Air" vs. "Dense Tissue" were set using the ROI and histogram tools. For total tumor burden analyses, an object map was created using the previously established thresholds and manually adjusted using "Spline Edit", "Draw", "Trace", and "Nudge Edit" tools. The object map was then morphed, i.e., made binary by using the threshold morphing tool. Then the map was dilated 3 times using $5 \times 5 \times 5$ Jack-shaped structuring elements. Holes were filled on every two-dimensional orientation and the map was finally brought back to its original size with the "Erode" tool 3 times using $5 \times 5 \times 5$ Jack-shaped structuring elements. The volumetric analyses were then performed in the ROI window using the pre-established thresholds and non-airspace was calculated using the formula: Nonairspace $=1-$ (VolAir/ROIVol).

Immunohistochemistry. Tissues were fixed in formalin overnight, then transferred to $70 \%$ ethanol, and embedded in paraffin (ARUP histology core). Formalin-fixed paraffin-embedded sections (4 micron) were used for hematoxylin and eosin and IHC staining. Antigen retrieval was performed by boiling slides for $20 \mathrm{~min}$ in $0.01 \mathrm{M}$ citrate buffer, $\mathrm{pH}$ 6.0. Slides were blocked for $15 \mathrm{~min}$ with $3 \% \mathrm{H}_{2} \mathrm{O}_{2}$, followed by $5 \%$ goat serum in PBS containing $0.1 \%$ Tween- 20 (PBST). Primary antibodies were incubated overnight at $4{ }^{\circ} \mathrm{C}$ and include the following: BCL2 (\#M088701-2, clone 124, Agilent), MYC (ab32072, Abcam), NEUROD1 (ab205300, Abcam), and ASCL1 (\#556604, BD Pharmingen). Slides were then incubated with horseradish peroxidase (HRP)-conjugated secondary antibody (Vector Laboratories, 1:200) and developed with DAB (Vector Laboratories). A Nikon Eclipse Ci microscope and DS-Fi3 camera were used for imaging.

Immunoblot. Cell lysates were prepared using RIPA buffer supplemented with protease inhibitors (cOmplete Mini Protease Inhibitor Cocktail, Roche). Protein concentration was determined by BCA assay (Pierce) and equal amounts of protein were separated on 4-20\% Tris-glycine sodium dodecyl sulfate-polyacrylamide gel electrophoresis (SDS-PAGE) gels (Thermo Fisher Scientific) and transferred to PVDF-FL membrane (Millipore). Membranes were blocked in 3\% cold-fish gelatin blocking buffer in TBS, incubated with primary antibodies, washed, and incubated with fluorescently labeled secondary antibodies prior to detection with Odyssey CLx imaging system (LI-COR Biosciences). Images were processed using the Image Studio Software (LI-COR Biosciences). Primary antibodies are: MYC (\#9402, Cell Signaling Technology, 1:1000), MYCN (sc-53993, Santa Cruz Biotechnology, 1:1000), MYCL (AF4050, R\&D Systems, 1:1000), BCL2 (\#2872, Cell Signaling Technology, 1:1000),
BIM (\#2933, Cell Signaling Technology, 1:1000), BAD (\#610391, BD Biosciences, 1:1000), BCL-XL (\#2764, Cell Signaling Technology, 1:1000), HA (\#3724, Cell Signaling Technology, 1:1000), MIZ1 (clone 10E2, Elmar Wolf, Würzburg, 1:500), ASCL1 (\#556604, BD Biosciences, 1:1000), MCL1 (sc-819, Santa Cruz Biotechnology, 1:1000), $\gamma$ H2AX (\#05-636, Merck, 1:1000), Cleaved Caspase 3 - CC3 (\#9664, Cell Signaling Technology, 1:500), pAURKA/B/C (\#2914, Cell Signaling Technology, $1: 1000)$, $\mathrm{pCHK}^{\mathrm{S} 345}$ (\#2341, Cell Signaling Technology, 1:1000), and HSP90 (ADISPA-835, Enzo Life Sciences, 1:5000). Secondary antibodies are: goat anti-rabbit 800CW (\#926-32211, LI-COR Biosciences, 1:10,000), goat anti-mouse 800CW (\#9263220, LI-COR Biosciences, 1:10,000), anti-rat 680 (\#925-68029, LI-COR Biosciences, 1:10,000), goat anti-rabbit 680LT (\#926-68021, LI-COR Biosciences, 1:10,000), and goat anti-mouse 680LT (\#926-68020, LI-COR Biosciences, 1:10,000). Alternatively, cells were lysed in RIPA buffer supplemented with Pierce Protease inhibitors and sodium orthovanadate. Protein concentrations were measured with the DC protein assay (Bio-Rad), and equal protein volumes were resolved on SDS-PAGE gels. Samples were transferred to $0.2 \mu \mathrm{m}$ PVDF (Bio-Rad). Membranes were blocked in 5\% milk/PBS-T prior to overnight incubation in primary antibody. Membranes were then incubated in secondary anti-rabbit-HRP or anti-mouse-HRP antibody (Jackson ImmunoResearch, 1:4000). After washing, membranes were developed with WesternBright ECL HRP (Advansta) and imaged on Hyblot autoradiography film. Primary antibodies used include the following: BCL2 (\#2872, Cell Signaling Technology, 1:2000); MCL1 (\#94296, Cell Signaling Technology, 1:2000); HSP90 (\#4877, Cell Signaling Technology, 1:2000); and ACTIN (\#A2066, Sigma, 1:10,000). Uncropped blots are displayed in the Source Data file.

Reporting summary. Further information on research design is available in the Nature Research Reporting Summary linked to this article.

\section{Data availability}

All data supporting the findings in this study are available from the corresponding author upon reasonable request. The primary data underlying the graphs are provided in the Source Data File. Previously published datasets used in this study are available at Gene Expression Omnibus through accession codes GSE60052 (expression data SCLC patients) and GSE68379 (methylation data) and at European Genome-phenome Archive through accession codes EGAS00001002115 and EGAS00001000334 (both RNAseq human SCLC cell lines). RNAseq and WES data generated in this study have been deposited at EBI Array Express with the accession codes E-MTAB-7410 (RNAseq NCI-H69, COR-L303), E-MTAB-7411 (RNAseq rp181 CRISPRa), and E-MTAB-7412 (WES rp181).

Received: 30 November 2018 Accepted: 10 July 2019

Published online: 02 August 2019

\section{References}

1. George, J. et al. Comprehensive genomic profiles of small cell lung cancer. Nature 524, 47-53 (2015).

2. Peifer, M. et al. Integrative genome analyses identify key somatic driver mutations of small-cell lung cancer. Nat. Genet. 44, 1104-1110 (2012).

3. Gustafson, W. C. et al. Drugging MYCN through an allosteric transition in aurora kinase A. Cancer Cell 26, 414-427 (2014).

4. Dauch, D. et al. A MYC-aurora kinase A protein complex represents an actionable drug target in p53-altered liver cancer. Nat. Med. 22, 744-753 (2016).

5. Beaulieu, M. E. et al. Intrinsic cell-penetrating activity propels omomyc from proof of concept to viable anti-myc therapy. Sci. Transl. Med. 11, 1-14 (2019).

6. Mollaoglu, G. et al. MYC drives progression of small cell lung cancer to a variant neuroendocrine subtype with vulnerability to aurora kinase inhibition. Cancer Cell 31, 270-285 (2017).

7. Sos, M. L. et al. A framework for identification of actionable cancer genome dependencies in small cell lung cancer. Proc. Natl Acad. Sci. USA 109, 17034-17039 (2012)

8. Cardnell, R. J. et al. Protein expression of TTF1 and cMYC define distinct molecular subgroups of small cell lung cancer with unique vulnerabilities to aurora kinase inhibition, DLL3 targeting, and other targeted therapies. Oncotarget 8, 73419-73432 (2017).

9. Sen, T. et al. CHK1 inhibition in small-cell lung cancer produces single-agent activity in biomarker-defined disease subsets and combination activity with cisplatin or olaparib. Cancer Res. 77, 3870-3884 (2017).

10. Faber, A. C. et al. Assessment of ABT-263 activity across a cancer cell line collection leads to a potent combination therapy for small-cell lung cancer. Proc. Natl Acad. Sci. USA 112, E1288-E1296 (2015).

11. Rudin, C. M. et al. Comprehensive genomic analysis identifies SOX2 as a frequently amplified gene in small-cell lung cancer. Nat. Genet. 44, 1111-1116 (2012).

12. Johnson, B. E. et al. Myc family oncogene amplification in tumor cell lines established from small cell lung cancer patients and its relationship to clinical status and course. J. Clin. Invest. 79, 1629-1634 (1987). 
13. Konermann, S. et al. Genome-scale transcriptional activation by an engineered CRISPR-Cas9 complex. Nature 517, 583-8 (2014).

14. Meuwissen, R. et al. Induction of small cell lung cancer by somatic inactivation of both Trp53 and Rb1 in a conditional mouse model. Cancer Cell 4, 181-189 (2003).

15. Jiang, L. et al. Genomic landscape survey identifies SRSF1 as a key oncodriver in small cell lung cancer. PLoS Genet. 12, 1-22 (2016).

16. Barretina, J. et al. The Cancer Cell Line Encyclopedia enables predictive modelling of anticancer drug sensitivity. Nature 483, 603-607 (2012)

17. Stransky, N. et al. Pharmacogenomic agreement between two cancer cell line data sets. Nature 528, 84-87 (2015).

18. Zhang, W. et al. Small cell lung cancer tumors and preclinical models display heterogeneity of neuroendocrine phenotypes. Transl. Lung Cancer Res. 7, 32-49 (2018)

19. Lochmann, T. L. et al. Venetoclax is effective in small cell lung cancers with high BCL-2 expression. Clin. Cancer Res. 24, 360-369 (2018).

20. Iorio, F. et al. A landscape of pharmacogenomic interactions in cancer. Cell 166, 740-754 (2016).

21. Poirier, J. T. et al. DNA methylation in small cell lung cancer defines distinct disease subtypes and correlates with high expression of EZH2. Oncogene 34, 5869-5878 (2015).

22. Brenner, C. et al. Myc represses transcription through recruitment of DNA methyltransferase corepressor. EMBO J. 24x2, 336-346 (2005).

23. Vo, B. H. T. et al. The interaction of Myc with Mizl defines medulloblastoma subgroup identity. Cancer Cell 29, 5-16 (2016).

24. Patel, J. H. \& Mcmahon, S. B. BCL2 is a downstream effector of MIZ-1 essential for blocking c-MYC-induced apoptosis. J. Biol. Chem. 282, 5-13 (2007).

25. Deng, J. et al. BH3 profiling identifies three distinct classes of apoptotic blocks to predict response to ABT-737 and conventional chemotherapeutic agents. Cancer Cell 12, 171-185 (2007).

26. Kotschy, A. et al. The MCL1 inhibitor S63845 is tolerable and effective in diverse cancer models. Nature 538, 477-482 (2016).

27. Brennan, M. S. et al. Humanized Mcl-1 mice enable accurate pre-clinical evaluation of MCL-1 inhibitors destined for clinical use. Blood 129, 846-854 (2018).

28. Harley, M. E., Allan, L. A., Sanderson, H. S. \& Clarke, P. R. Phosphorylation of Mcl-1 by CDK1-cyclin B1 initiates its Cdc20-dependent destruction during mitotic arrest. $E M B O$ J. 29, 2407-2420 (2010).

29. Strasser, A., Harris, A. W., Jacks, T. \& Cory, S. DNA damage can induce apoptosis in proliferating lymphoid cells via p53-independent mechanisms inhibitable by Bcl-2. Cell 79, 329-339 (1994).

30. Ichim, G. et al. Limited mitochondrial permeabilization causes DNA damage and genomic instability in the absence of article limited mitochondrial permeabilization causes DNA damage and genomic instability in the absence of cell death. Mol. Cell 57, 860-872 (2015).

31. Liewer, S. \& Huddleston, A. Alisertib: a review of pharmacokinetics, efficacy and toxicity in patients with hematologic malignancies and solid tumors. Expert Opin. Investig. Drugs 27, 105-112 (2018).

32. Owonikoko, T. et al. OA05.05 randomized phase 2 study: alisertib (MLN8237) or placebo + paclitaxel as second-line therapy for small-cell lung cancer (SCLC). J. Thorac. Oncol. 12, S261-S262 (2017).

33. Owonikoko, T. K. et al. Randomized phase 2 study of investigational aurora A kinase (AAK) inhibitor alisertib (MLN8237) + paclitaxel (P) vs placebo + P as second line therapy for small-cell lung cancer (SCLC). Ann. Oncol. 27, 493-6 (2016)

34. Cun, Y., Yang, T. P., Achter, V., Lang, U. \& Peifer, M. Copy-number analysis and inference of subclonal populations in cancer genomes using Sclust. Nat. Protoc. 13, 1488-1501 (2018).

35. Dobin, A. et al. STAR: ultrafast universal RNA-seq aligner. Bioinformatics 29, 15-21 (2013)

36. Li, B. \& Dewey, C. N. RSEM: accurate transcript quantification from RNASeq data with or without a reference genome. BMC Bioinformatics 12, 323 (2011).

37. Love, M. I., Huber, W. \& Anders, S. Moderated estimation of fold change and dispersion for RNA-seq data with DESeq2. Genome Biol. 15, 550 (2014).

38. Ingaramo, M. et al. Two-photon excitation improves multifocal structured illumination microscopy in thick scattering tissue. Proc. Natl Acad. Sci. USA 111, 5254-5259 (2014).

39. Schaffer, B. E. et al. Loss of p130 accelerates tumor development in a mouse model for human small-cell lung carcinoma. Cancer Res. 70, 3877-3883 (2010).

40. Tian, Y. et al. ChAMP: updated methylation analysis pipeline for Illumina BeadChips. Bioinformatics 33, 3982-3984 (2017).
41. Dedeurwaerder, S. et al. Evaluation of the Infinium Methylation $450 \mathrm{~K}$ technology. Epigenomics 3, 771-784 (2011).

42. He, L. et al. Methods for high-throughput drug combination screening and synergy scoring. Methods Mol. Biol. 1711, 351-398 (2018).

43. Kumaki, Y., Oda, M. \& Okano, M. QUMA: quantification tool for methylation analysis. Nucleic Acids Res. 36, 170-175 (2008).

\section{Acknowledgements}

We thank Marek Franitza, Christian Becker, and Janine Altmüller (Cologne Center for Genomics, University of Cologne) for technical support and Katia Garbert, Christian Müller, and Graziella Bosco (University of Cologne) for assistance with data acquisition. We also thank members of the Oliver Laboratory for technical support, especially Ismail Can and Danny Soltero. T.G.O. was supported in part by the NIH NCI (R01CA187487 and R21CA216504), the American Lung Association (LCD-506758), and the Huntsman Cancer Institute from the NIH NCI (P30CA042014). This work was furthermore supported by the Deutsche Forschungsgemeinschaft (CRC1399 to R.B., R.K.T., H.C.R., M.L.S.; KFO-286/RP2, RE 2246/2-1, RE 2246/7-1 to H.C.R. and TH1386/3-1 to R.K.T., M.L.S.), the Bundesministerium für Bildung und Forschung (e:Med 01ZX1603A to H.C.R., R.B., R.K.T. and $01 Z X 1406$ to M.L.S.), the German federal state North Rhine Westphalia (NRW) as part of the EFRE initiative (EFRE-0800397 to H.C.R., R.B., R.K.T. and M.L.S.), the Else KrönerFresenius Stiftung (EKFS-2014-A06 and 2016_Kolleg.19 to H.C.R., R.C., R.D.J., S.K. and Memorial Grant 2018_EKMS.35 to J.B.), the Köln Fortune program (to A.M.), and the Deutsche Krebshilfe (70113041 to H.C.R., 70112888 to M.L.S. and 70113129 to C.L.) Additional funding was provided by the Deutsche Krebshilfe as part of the Oncology Centers of Excellence funding program (to R.B.) and as part of the Small Cell Lung Cancer Genome Sequencing Consortium (109679 to R.K.T., R.B.).

\section{Author contributions}

M.A.D., J.B., R.R.O., S.B., N.M., C.P.W., M.D.C., H.L.T., M.R.G., S.K., A.S.I., A.M., J.R., L.O., R.C., C.L., T.S. and J.T.P. performed experiments and analyzed and visualized the data; M.A.D., J.B., R.R.O., S.B., T.S., L.A.B., A.L., J.T.P., T.G.O. and M.L.S. conceived experimental design; A.S., R.D.J., E.W., R.K.T., H.C.R. and R.B., provided resources; M.A.D., J.B., S.B., T.G.O. and M.L.S. wrote the manuscript; T.G.O. and M.L.S. conceived the study, acquired funding, and supervised the project.

\section{Additional information}

Supplementary Information accompanies this paper at https://doi.org/10.1038/s41467 019-11371-x.

Competing interests: M.L.S. and R.K.T. are founders and shareholders of PearlRiver Bio M.L.S. received a commercial research grant from Novartis. R.K.T. is a founder of NEO New Oncology GmbH, now part of Siemens Healthcare, and received consulting and lecture fees from Merck, Roche, Lilly, Boehringer Ingelheim, Astra-Zeneca, DaiichiSankyo, MSD, NEO New Oncology, Puma, and Clovis. H.C.R. received consulting and lecture fees from Abbvie, Astra-Zeneca, Vertex, and Merck and received research funding from Gilead Pharmaceuticals; R.B. is an employee of Targos Molecular Pathology. The other authors declare no competing interests.

Reprints and permission information is available online at http://npg.nature.com/ reprintsandpermissions/

Peer review information: Nature Communications thanks the anonymous reviewers for their contribution to the peer review of this work.

Publisher's note: Springer Nature remains neutral with regard to jurisdictional claims in published maps and institutional affiliations.

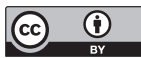

Open Access This article is licensed under a Creative Commons Attribution 4.0 International License, which permits use, sharing, adaptation, distribution and reproduction in any medium or format, as long as you give appropriate credit to the original author(s) and the source, provide a link to the Creative Commons license, and indicate if changes were made. The images or other third party material in this article are included in the article's Creative Commons license, unless indicated otherwise in a credit line to the material. If material is not included in the article's Creative Commons license and your intended use is not permitted by statutory regulation or exceeds the permitted use, you will need to obtain permission directly from the copyright holder. To view a copy of this license, visit http://creativecommons.org/ licenses/by/4.0/

(C) The Author(s) 2019 